\title{
Influence of a tight isotropic harmonic trap on photoassociation in ultracold homonuclear alkali gases
}

\author{
Sergey Grishkevich and Alejandro Saenz \\ AG Moderne Optik, Institut für Physik, Humboldt-Universität zu Berlin, Hausvogteiplatz 5-7, 10117 Berlin, Germany
}

(Dated: October 5, 2018)

\begin{abstract}
The influence of a tight isotropic harmonic trap on photoassociation of two ultracold alkali atoms forming a homonuclear diatomic is investigated using realistic atomic interaction potentials. Confinement of the initial atom pair due to the trap leads to a uniform strong enhancement of the photoassociation rate to most, but also to a strongly suppressed rate for some final states. Thus tighter traps do not necessarily enhance the photoassociation rate. A further massive enhancement of the rate is found for strong interatomic interaction potentials. The details of this interaction play a minor role, except for large repulsive interactions for which a sharp window occurs in the photoassociation spectrum as is known from the trap-free case. A comparison with simplified models describing the atomic interaction like the pseudopotential approximation shows that they often provide reasonable estimates for the trap-induced enhancement of the photoassociation rate even if the predicted rates can be completely erroneous.
\end{abstract}

\section{INTRODUCTION}

Over the past ten years there has been an increasing interest in ultracold atomic and molecular physics. This interest was stimulated by the successful experimental observation of Bose-Einstein condensation (BEC) in dilute atomic gases [1]. These atomic condensates exhibit many qualitatively new features. Besides their relevance to fundamental quantum statistical and possibly even solid-state questions a further interesting aspect is that the atoms can bind together to form ultracold and even Bose-Einstein condensed molecules 2, 3, 4].

Although so far the only successful way for achieving a molecular BEC is based on magnetic Feshbach resonances, alternative schemes are still highly desirable, since magnetic Feshbach resonances do not appear to be a universal tool. One of the alternative schemes is photoassociation where two ultracold or Bose-condensed atoms absorb a photon and form a bound excited molecule [5, 6]. Although it was demonstrated that this process generates cold molecules, the yield is small compared to the one obtained by means of magnetic Feshbach resonances. The advantage of photoassociation (and related coherentcontrol schemes) compared to Feshbach resonances is, however, their assumed wider range of applicability, since there is no need for the occurrence of suitable resonances and thus no requirement for specific magnetic properties of the atoms involved.

Besides simple one-step photoassociation that yields electronically excited molecules there are also resonant or non-resonant multi-step schemes leading to the electronic and possibly even rovibrational ground state. One of the schemes to produce molecules from atoms with help of lasers is, e. g., two-color stimulated Raman adiabatic passage 7]. The present work discusses only one-photon association explicitly, but it is important to note that the discussed transition matrix elements are direct ingredients for the modeling of more sophisticated schemes like the mentioned two-photon processes.

Photoassociation is also a powerful tool for the inves- tigation of the properties of cold atoms and diatomic molecules. The absorption of the photon typically occurs at large internuclear distances, and thus the photoassociation spectrum provides important information about the long-range part of the molecular potential curves as well as the collisional properties of atoms [8, 9, 10, 11].

The cooling of atomic samples is usually achieved in a trap and thus photoassociation experiments in ultra-cold atomic gases are performed in the presence of a trap potential. In most cases these traps are rather shallow so that the corresponding trap frequency $\omega$ is of the order of $100 \mathrm{~Hz}$ [12]. (Here and in the following the frequency of the trap corresponds to the one in the harmonic approximation). For such a frequency the influence of the trap is expected to be negligible. This may, however, change for very tight traps. In fact, it was pointed out that the atom-molecule conversion process is more efficient, if photoassociation is performed under tight trapping conditions as they are, e.g., accessible in optical lattices [13]. The advantage of using tight confinement has stimulated further theoretical investigations and very recently some proposals were made that discuss the possibility of using the trapping potential itself for the formation of molecules [14, 15].

The study of photoassociation in tight optical lattices is of interest by itself, since it is possible to achieve tailored Mott-Insulator states containing a large number of almost identical lattice sites, each filled with exactly two atoms 16]. The trap frequency of a lattice site in which molecules are produced via photoassociation can be of the order of $100 \mathrm{kHz}$ [17]. The systematic investigation of the influence of a tight isotropic harmonic trap on the photoassociation process of two alkali atoms forming a homonuclear molecule is the topic of this work. Realistic atom-atom interaction potentials are adopted. This allows to check also the range of applicability of the $\delta$-function (pseudopotential) approximation for the description of the photoassociation process.

In the pseudopotential approximations the true atomatom interaction is replaced by one that reproduces 
asymptotically the two-body zero-energy s-wave scattering. For this choice of the potential ( $\delta$-function) and if the two atoms are placed in a harmonic trap the Schrödinger equation possesses an analytical solution [18, 19]. The validity regime of the pseudopotential approximation has been discussed with respect to the energy levels for trapped atoms in [20]. It was shown that the use of an energy-dependent pseudopotential (instead of the mostly adopted energy-independent one) gives almost correct energy levels for two harmonically trapped atoms. Whether this simplified model for the atomic interaction is appropriate for the description of photoassociation in a harmonic trap is, however, not immediately evident. Therefore, the present work compares the results obtained using realistic atomic interaction potentials with the ones obtained with either the energydependent or -independent pseudopotential.

Photoassociation in tight traps has been studied theoretically before 21]. The energy-independent pseudopotential approximation was adopted and only photoassociation into long-range states discussed. Since the present work uses realistic atomic interaction potentials, transitions to all final vibrational states can be considered. This allows to identify two different regimes with respect to the influence of a tight trap on the photoassociation rate as well as (approximate) rules where a transition from one regime to the other is to be expected.

The outline of this work is the following. First, a brief description of the model systems is given in Sec.III In Sec.III the influence of a tight trap on photoassociation is discussed. This includes after a brief general discussion of the trap influence in Sec.IIIA the derivation of a sum rule in Sec.IIIB the introduction of an enhancement or suppression factor in Sec. IIIC and the discussion of two regimes in Sec.【IID and IIIE Then the case of repulsive atom-atom interactions is considered in Sec.【IF The combined influence of trap frequency and atom-atom interaction on photoassociation is investigated in Sec.IIG the validity of the pseudopotential approximation in Sec.IV Finally, a discussion and outlook is given in Sec. $\mathbf{D}$ All equations and quantities in this paper are given in atomic units unless otherwise specified.

\section{THE SYSTEM}

Photoassociation of two identical atoms confined in an isotropic harmonic trap and interacting through a twobody Born-Oppenheimer potential $V_{\text {int }}(R)$ is considered. The spherical symmetry and harmonicity of the trap allows to separate the center-of-mass and the radial internal motion [18]. The eigenfunctions of the center-of-mass motion are the harmonic-oscillator states. Thus the problem reduces to solving the Schrödinger equation for the radial internal motion

$$
\begin{aligned}
{\left[\frac{1}{2 \mu} \frac{d^{2}}{d R^{2}}-\frac{J(J+1)}{2 \mu R^{2}}\right.} & -V_{\mathrm{int}}(R)-\frac{1}{2} \mu \omega^{2} R^{2} \\
& +E] \Psi(R)=0 .
\end{aligned}
$$

In Eq. (11) $J$ denotes the rotational quantum number, $\omega$ is the harmonic trap frequency, and $\mu$ is the reduced mass that is equal to $m / 2$ in the present case of particles with identical mass $m$.

In order to compute the photoassociation spectrum the vibrational wave functions $\Psi(R) / R$ are determined for the initial and final molecular states from Eq. (10 with the corresponding Born-Oppenheimer interaction potentials $V_{\text {int }}(R)$. The equation is solved numerically using an expansion in $B$ splines. For the investigation of the influence of the trap on the photoassociation rate Eq. (10) is solved for $\omega \neq 0$.

The photoassociation processes most relevant to experiments on ultracold alkali atoms correspond to transitions from two free ground-state atoms (interacting via the ground triplet or singlet potential) to the different vibrational levels of the first excited triplet or singlet state 22, 23, 24]. Due to hyperfine interaction, two alkali atoms can also interact via a coherent admixture of singlet and triplet states. This work starts by considering the photoassociative transition between the two triplet states $a^{3} \Sigma_{u}^{+}$and $1^{3} \Sigma_{g}^{+}$for ${ }^{6} \mathrm{Li}$. A corresponding experiment is, e. g., reported in 12. The generality of the conclusions drawn from this specific example are then tested by considering also other atoms $\left({ }^{7} \mathrm{Li}\right.$ and $\left.{ }^{39} \mathrm{~K}\right)$ or modifying artificially the interaction strength, as is discussed below.

For the short-range part of the $a^{3} \Sigma_{u}^{+}$molecular potential of $\mathrm{Li}_{2}$ the data in [25] are used, including the van der Waals coefficients cited therein. In the case of the $1^{3} \Sigma_{g}^{+}$ state data for interatomic distances between $R=4.66 a_{0}$ and $R=7.84 a_{0}$ are taken from [26] and are extended with $a b$ initio values from 27] for distances between $R=3.25 a_{0}$ and $R=4.50 a_{0}$ and between $R=8.0 a_{0}$ and $R=30.0 a_{0}$. The van der Waals coefficients from 28] are used. For a $\Sigma$ to $\Sigma$ molecular dipole transition the selection rule is $J=J^{\prime} \pm 1$. Assuming ultracold atomic gases the atoms interact initially in the $J^{\prime}=0$ state of the $a^{3} \Sigma_{u}^{+}$potential. The dipole selection rule leads then to transitions to the $J=1$ states of $1^{3} \Sigma_{g}^{+}$. With the given potential-curve parameters a solution of Eq. (11) in the absence of a trap $(\omega=0)$ yields for the fermionic ${ }^{6} \mathrm{Li}$ atoms 10 and 100 vibrational bound states for the $a^{3} \Sigma_{u}^{+}$ $\left(J^{\prime}=0\right)$ and the $1^{3} \Sigma_{g}^{+}(J=1)$ states, respectively. In the case of the bosonic ${ }^{7} \mathrm{Li}$ atoms there are 11 and 108 vibrational bound states for the $a^{3} \Sigma_{u}^{+}\left(J^{\prime}=0\right)$ and the $1^{3} \Sigma_{g}^{+}(J=1)$ states, respectively.

The electronic dipole moment $D(R)$ for the transition $a^{3} \Sigma_{u}^{+} \rightarrow 1^{3} \Sigma_{g}^{+}$of Li was calculated [29] with a configuration interaction (CI) method for the two valence electrons using the code described in 30 . The core electrons were described with the aid of the Klapish model 
potential with the parameters given in 31] and polarization was considered as discussed in [32]. The resulting $D(R)$ (and its value in the separated atom limit) is in good agreement with literature data 27, 28, 33, 34].

In the limit of zero collision energy the interaction between two atoms can be characterised by their s-wave scattering length $a_{\mathrm{sc}}$. Its sign determines the type of interaction (repulsive or attractive) and its absolute value the interaction strength. For a given potential curve the s-wave $\left(J^{\prime}=0\right)$ scattering length can be determined using the fact that at large distances the scattering wave function describing the relative motion (for $\omega=0$ and very small collision energies) reaches an asymptotic behavior of the form

$$
\Psi_{E}(R)=\sqrt{\frac{k}{\pi E}} \sin \left[k\left(R-a_{\mathrm{sc}}\right)\right] .
$$

In the present numerical approach discretised continuum states are obtained, since the wave-function calculation is performed within a finite $R$ range, i.e. in the interval $\left[0, R_{\max }\right]$. Only wave functions that decay before or have a node at $R_{\max }$ are obtained. From the analysis of the lowest lying discretised continuum state the scattering length $a_{\mathrm{sc}}$ is obtained by the use of the relation $a_{\mathrm{sc}}=R_{\max }-\frac{\pi}{k}$ with $k=\sqrt{2 \mu E}[35]$. A variation of $R_{\max }$ changes the continuum discretization and therefore results in $R_{\max }$-dependent lowest lying continuum solutions $\Psi_{E_{0}}$. The scattering length extracted from $\Psi_{E_{0}}$ converges, however, to a constant value as $R_{\max }$ increases and $E_{0}$ approaches zero. Using this method and the adopted potential curves the scattering length values $a_{\mathrm{sc}}=-2030 a_{0}$ and $a_{\mathrm{sc}}=-30 a_{0}$ are obtained for ${ }^{6} \mathrm{Li}$ and ${ }^{7} \mathrm{Li}$, respectively. These values agree well with the experimental ones: $a_{\mathrm{sc}}=(-2160 \pm 250) a_{0}\left({ }^{6} \mathrm{Li}\right)$ and $a_{\mathrm{sc}}=(-27.6 \pm 0.5) a_{0}\left({ }^{7} \mathrm{Li}\right)[36]$.

The interaction of two ultracold ${ }^{6} \mathrm{Li}$ atoms is strongly, the one of ${ }^{7} \mathrm{Li}$ weakly attractive, as is reflected by the large and small but negative scattering lengths. In the case of two identical fermionic ${ }^{6} \mathrm{Li}$ atoms the asymmetry requirement of the total wave function excludes s-wave scattering. Thus the present results are more applicable for two ${ }^{6} \mathrm{Li}$ atoms in different hyperfine states (where the admixture of a singlet potential would, however, usually modify the scattering length), but are actually meant as a realistic example for a very large negative scattering length, i.e. strong attraction. In order to further check the generality of the results also the formation of ${ }^{39} \mathrm{~K}_{2}$ is investigated as an example for a small repulsive interaction. In this case photoassociation starting from two potassium atoms interacting via the singlet $X^{1} \Sigma_{g}^{+}$ground state and transitions into the $A^{1} \Sigma_{u}^{+}$state are considered. This process is not only experimentally relevant [37], but is at the same time an even further check of the generality of the conclusions obtained from the investigation of the transitions between triplet states in $\mathrm{Li}_{2}$.

The data for constructing the relevant potential curves for ${ }^{39} \mathrm{~K}_{2}$ are taken from [38, 39, 40]. The resulting potential curve for the $X^{1} \Sigma_{g}^{+}$state yields a scattering length $a_{\mathrm{sc}} \approx+90 a_{0}$. This is in reasonable agreement with the experimental value given in [41] where $a_{\mathrm{sc}}$ is found to be lying between $+90 a_{0}$ and $+230 a_{0}$.

Instead of selecting additional atomic pairs that could represent examples for other values of the scattering length, the sensitivity of the s-wave interaction on the position of the least bound state is used to generate artificially a variable interaction strength. The scattering length is thus modified by a variation of the particle mass. The strong mass dependence of the scattering length is already evident from its change from $-2030 a_{0}$ to $-30 a_{0}$ for the isotopes ${ }^{6} \mathrm{Li}$ and ${ }^{7} \mathrm{Li}$, respectively. Experimentally, a strong variation of the interaction strength can be realized by the aid of magnetic Feshbach resonances [42, 43].

\section{PHOTOASSOCIATION IN AN ISOTROPIC HARMONIC TRAP}

\section{A. Photoassociation in a trap}

While Eq. (11) yields in the trap-free case $(\omega=0)$ both bound (vibrational) and continuum (dissociative) states, the harmonic trap potential changes the energy spectrum to a purely discrete one, as is sketched in Fig. 10 Considering the concrete example of two ${ }^{6} \mathrm{Li}$ atoms where

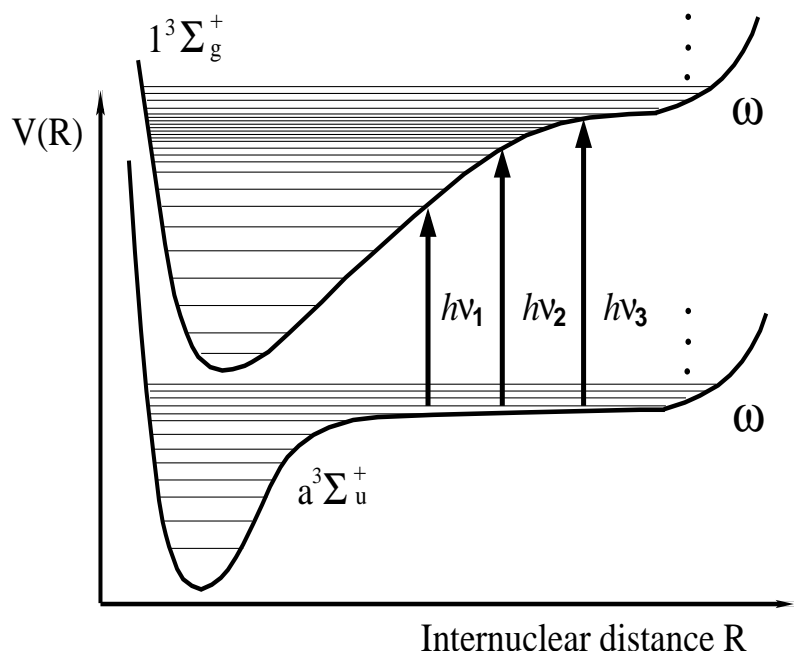

FIG. 1: Sketch of the photoassociation process for ${ }^{6} \mathrm{Li}_{2}$ in the presence of a trap (not to scale). With the aid of a laser photoassociation is induced from the first trap-induced bound state $\left(v^{\prime}=10\right)$ of the $a^{3} \Sigma_{u}^{+}$state into some vibrational level $v$ of the $1^{3} \Sigma_{g}^{+}$state. Different laser frequencies $\nu_{i}$ couple the same initial state to different final states.

the $a^{3} \Sigma_{u}^{+}$state supports the 10 vibrational bound states $v^{\prime}=0$ to $9, v^{\prime}=10\left(J^{\prime}=0\right)$ denotes the first state that results from the trap-induced continuum discretization. This (first trap-induced) state describes the initial state of two spin-polarized ${ }^{6} \mathrm{Li}$ atoms interacting via the $a^{3} \Sigma_{u}^{+}$potential curve, if a sufficiently cold atomic gas 
in an (adiabatically turned-on) harmonic trap is considered. In the present work photoassociation (by means of a suitably tuned laser) from this initial state to one of the vibrational states $v$ of the $1^{3} \Sigma_{g}^{+}$potential is investigated as a function of the trap frequency $\omega$. In view of the already discussed relevant dipole-selection rule the final state possesses $J=1$ and in the following $J^{\prime}=0$ and $J=1$ is tacitly assumed. The strength of the photoassociation transition to final state $v$ is given by the rate [44]

$$
\Gamma_{v}(\omega)=4 \pi^{2} \mathcal{I} I^{v}(\omega)
$$

where $\mathcal{I}$ is the laser intensity and

$$
I^{v}(\omega)=\left|\int_{0}^{\infty} \Psi^{v}(R ; \omega) D(R) \Psi^{10^{\prime}}(R ; \omega) \mathrm{d} R\right|^{2} .
$$

In Eq. (4) $\Psi^{v}(R) / R$ and $\Psi^{10^{\prime}}(R) / R$ are the vibrational wave functions of the final and initial state, respectively. Since the radial density is proportional to $|\Psi|^{2}$, it is convenient to discuss $\Psi$ instead of the true vibrational wave function $\Psi(R) / R$. This will be done in the following where $\Psi$ is for simplicity called vibrational wave function. Finally, $D(R)$ is the ( $R$-dependent) electronic transition dipole matrix element between the $a^{3} \Sigma_{u}^{+}$and the $1^{3} \Sigma_{g}^{+}$ state of $\mathrm{Li}_{2}$ introduced in Sec.III $D(R)$ is practically constant for $R>25 a_{0}$. Eq. (4) is only valid within the dipole approximation. The latter is supposed to be applicable, if the photon wavelength is much larger than the extension of the atomic or molecular system. The shortest photoassociation laser wavelength corresponds to the transition to the highest-lying vibrational state and is thus approximately the one of the atomic $\left(2^{2} \mathrm{~S} \rightarrow 2^{2} \mathrm{P}\right.$ transition), $\lambda=12680 a_{0}$. Although the spatial extent of some of the final vibrational states (and of course the initial state in the case of shallow traps) has a similar or even larger extent, beyond dipole approximation effects are neglected in this work.

The key quantity describing the photoassociation rate to different vibrational states $v$ or for variable trap frequency $\omega$ is $I^{v}(\omega)$ on whose calculation and discussion this work concentrates. It is important to note that also in the case of more elaborate laser-assisted association schemes like stimulated Raman processes that involve (virtual) transitions to the $v$ states the transition rate is proportional to $I^{v}(\omega)$.

According to Eq. (4) the photoassociation rate depends for transitions between long-range states on the FranckCondon factors between the initial and final nuclear wave functions, if $D(R)$ is practically constant for large $R$. In the case of alkali atoms the interaction potentials of the electronic states can be very long ranged and can contain numerous rovibrational bound states. Fig. 2 shows, e.g., the classical outer turning points $R_{\text {out }}$ of the 100 $(J=0)$ vibrational bound states of ${ }^{6} \mathrm{Li}_{2}$ supported by the final-state electronic potential curve $1^{3} \Sigma_{g}^{+}$. The orthogonality of the states is achieved by the occurrence of

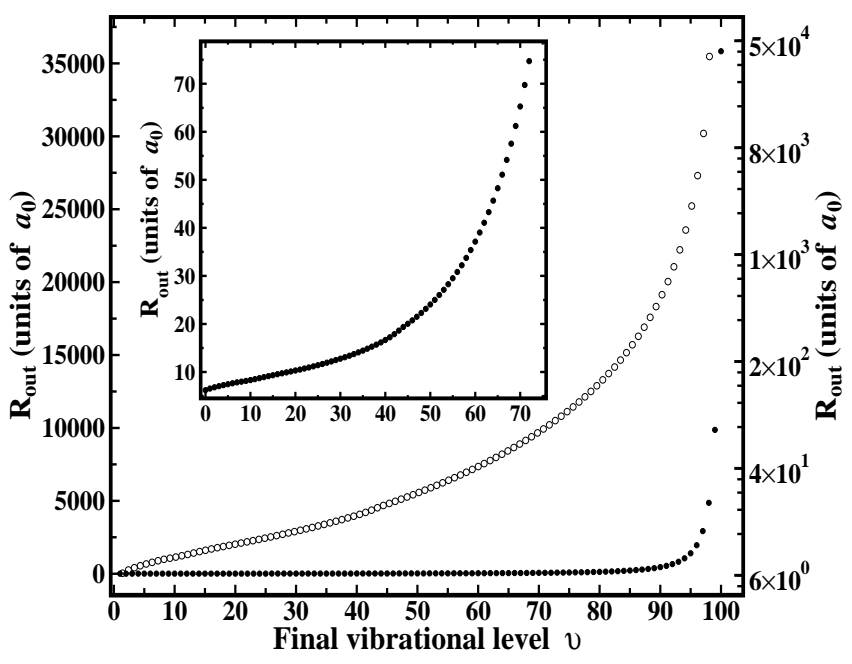

FIG. 2: The classical outer turning points of the vibrational levels of the $1^{3} \Sigma_{g}^{+}$state of ${ }^{6} \mathrm{Li}_{2}$ are shown on a linear (solid circles, left scale and insert) and on a logarithmic scale (empty circles, right scale).

$v^{\prime}$ nodes. As $v^{\prime}$ increases the wavefunctions consist of a highly oscillatory short range part with small overall amplitude that covers the range of the $v^{\prime}-1$ wavefunction and a large outermost lobe. The $1^{3} \Sigma_{g}^{+}$state is very long ranged, since its leading van der Waals term is $-C_{3} / R^{3}$. The initial electronic state $a^{3} \Sigma_{u}^{+}$with leading $-C_{6} / R^{6}$ van der Waals term is shorter ranged. Fig. 3 shows the initial vibrational state for ${ }^{6} \mathrm{Li}$ as a function of the trap frequency. This first trap-induced bound state possesses $v$ nodes (here $v=10$ ) that are located in the $R$ range of the last trap-free bound state $(v=9)$. The overall amplitude in this about $25 a_{0}$ long interval is very small and most of the wavefunction is distributed over the harmonic trap.

The squared transition dipole moments $I^{v}(\omega)$ are shown for ${ }^{6} \mathrm{Li}$ in Fig. 4(a) for three different trap frequencies $\omega$. As mentioned before, the final vibrational levels with $v>99$ are trap-induced bound states and exist only due to the continuum discretization in the presence of a trap. If the trap would be turned-off (adiabatically) after photoassociation to such a level, the trap induced dimer would immediately dissociate (without the need for any (radiative or non-radiative) coupling to some dissociative state).

For a fixed trap frequency the photoassociation rate generally increases as a function of the final vibrational level $v$, but for small $v$ an oscillatory behavior is visible. These oscillations are a consequence of the nodal structure of the initial-state wave functions describing the atom pair. The 10 nodes (for the shown example of ${ }^{6} \mathrm{Li}$ ) of the initial-state wave function lead to exactly 10 dips in photoassociation spectrum. Their exact position depends on the interference with the nodal structure of the final-state wave functions. The oscillatory structure of $I^{v}(\omega)$ ends at about $v=55$ and beyond that point 


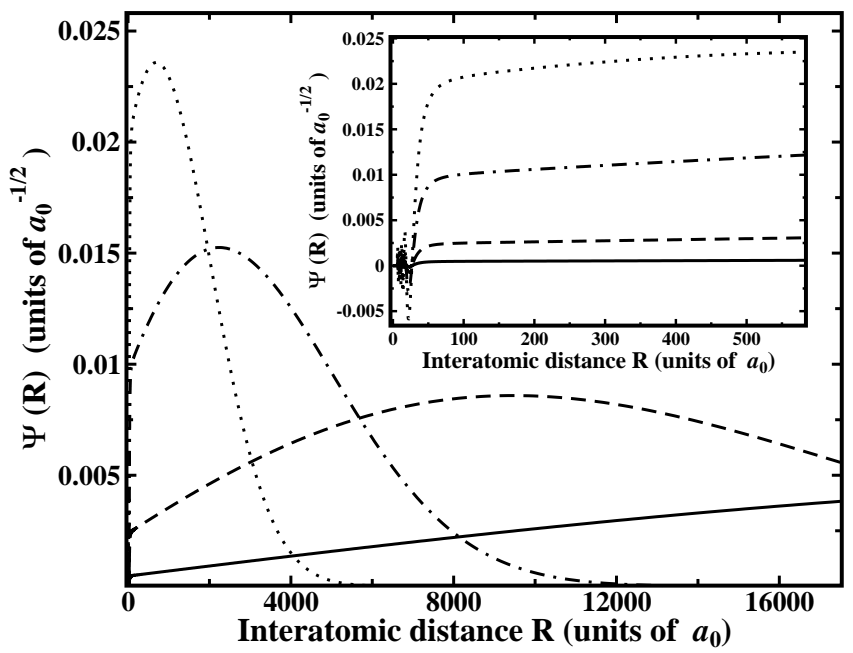

FIG. 3: Wave functions of the initial $a^{3} \Sigma_{u}^{+}$state of ${ }^{6} \mathrm{Li}_{2}$ for trap frequencies $\omega=2 \pi \times 1 \mathrm{kHz}$ (solid), $\omega=2 \pi \times 10 \mathrm{kHz}$ (dashes), $\omega=$ $2 \pi \times 100 \mathrm{kHz}$ (chain), and $\omega=2 \pi \times 500 \mathrm{kHz}$ (dots). (The insert shows the small $R$ range on an enlarged scale.)

the rate increases by orders of magnitude, before a sharp decrease is observed close to the highest lying vibrational bound state $(v=99)$. The absence of oscillatory behavior is a clear signature that for those transitions (in the present example for transitions into states with $v>55$ ) the Franck-Condon factors are determined by the overlap of the outermost lobe of the initial state with the one of the final state.

The comparison of $I^{v}(\omega)$ for the different trap frequencies shown in Fig. प(a) indicates a very systematic trend. The transition probabilities to most of the vibrational bound states increases with increasing trap frequency. This is in accordance with simple confinement arguments, since a tighter trap confines the atoms in the initial state to a smaller spatial region. Due to the special properties of harmonic traps, this confinement translates directly into a corresponding confinement of the pair density (see Eq.(10). The probability for atom pairs to have the correct separation for the photoassociative transition is thus expected to increase for tighter confinements, since a larger Franck-Condon overlap of the now more compact initial state with the bound molecular final state is expected. However, for the vibrational final states close to and above the (trap-free) dissociation threshold a completely different behavior is found. In this case the photoassociation rate decreases with increasing trap frequency, as can be seen especially from the insert of Fig. प(a). In fact, a sharp cut-off of the transition rate is observed. The transitions to the states that possessed the largest photoassociation rate for small trap frequencies are almost completely suppressed for large trap frequencies. Clearly, the simple assumption "a tighter trap leads to a higher photoassociation rate due to an increased spatial confinement" is only partly true. The fact that this assumption cannot be valid for all final states
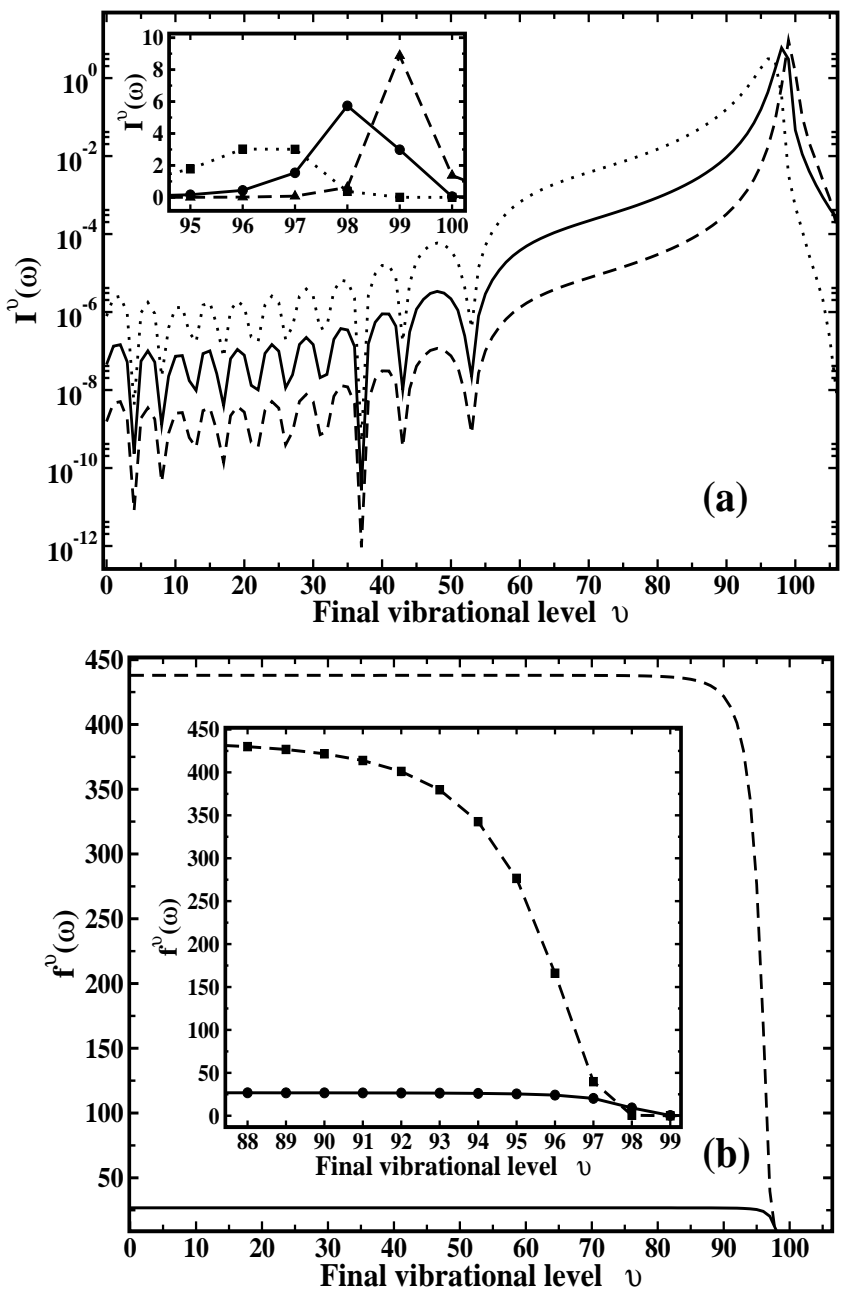

FIG. 4: (a) The squared dipole transition moments $I^{v}(\omega)$ describing transitions from the trap-induced $\left(v^{\prime}=10\right)$ initial $a^{3} \Sigma_{u}^{+}$state to the vibrational manifold $(v)$ of the $1^{3} \Sigma_{g}^{+}$state of ${ }^{6} \mathrm{Li}_{2}$ are shown for the trap frequencies $\omega=2 \pi \times 1 \mathrm{kHz}$ (dashes), $\omega=2 \pi \times 10 \mathrm{kHz}$ (solid), and $\omega=2 \pi \times 100 \mathrm{kHz}$ (dots). The insert shows the transitions to $v=95$ to 100 on an enlarged scale. For a better visibility the discrete transitions (marked explicitly with different symbols in the insert) are plotted as continuous lines. (b) The ratio $f^{v}(\omega)$ [defined in Eq. [6] is shown for $\omega=2 \pi \times 10 \mathrm{kHz}$ (solid) and $\omega=2 \pi \times 100 \mathrm{kHz}$ (dashes) as a function of the final vibrational level $v$. (As in (a) the discrete points are connected by lines to guide the eye.) The insert shows the transitions to $v=88$ to 99 on a magnified scale.

can be substantiated by means of a general sum-rule that is derived and discussed in the following subsection.

\section{B. Sum rule}

Performing a summation (including for $\omega=0$ an integration over the dissociative continuum) over all final 
vibrational states (using closure) yields

$$
\widetilde{I}(\omega)=\sum_{v=0}^{\infty} I^{v}(\omega)=\int_{0}^{\infty} \Psi^{10^{\prime}}(R ; \omega) D^{2}(R) \Psi^{10^{\prime}}(R ; \omega) \mathrm{d} R .
$$

While the electronic transition dipole moment $D(R)$ depends clearly on $R$ for small internuclear separations, it reaches its asymptotic value (the sum of the electronic dipole transition moments of two separated atoms, $D_{\text {at }}$ ) at some $R$ value that is much smaller than the typical spatial extend of the final vibrational states with the largest transition amplitudes. (In the example of $\mathrm{Li}_{2}$ this asymptotic limit is reached at about $25 a_{0}$.) If the largest photoassociation amplitudes result from transitions to final states whose wave functions are mostly located outside this $R$ range, the integral in Eq. (5]) is dominated by the $R$ regime in which $D(R)$ is constant. In this case $D^{2}$ can be taken out of the integral and normalization of the initial wave function assures $\widetilde{I}(\omega) \approx \widetilde{I}=D_{\text {at }}^{2}$.

Consequently, for all trap frequencies that are too small to confine the atoms into a spatial volume that is comparable to the atomic volumes (leading to $D(R) \neq$ $D_{\text {at }}$ ) and thus for all traps relevant to this work (and presently experimentally achievable) the total dipole transition moment $\widetilde{I}$ is to a good approximation independent of the trap frequency $\omega$. Therefore, changing the trap frequency can only redistribute transition probabilities between different final vibrational states. Increasing the transition rate to one final state must be compensated by a decrease of the transition probability to one or more other vibrational states.

A conservative estimate of the minimum and maximum influence of a harmonic trap on the photoassociation rate is obtained from $\widetilde{I}_{\min }=D_{\min }^{2}$ and $\widetilde{I}_{\max }=D_{\max }^{2}$, respectively, where $D_{\min }\left(D_{\max }\right)$ is the minimum (maximum) value of the molecular electronic transition dipole moment.

The sum-rule values obtained numerically for the trap frequencies shown in Fig. प4(a) are $\widetilde{I}(\omega=2 \pi \times 1 \mathrm{kHz})=$ $11.127222, \widetilde{I}(\omega=2 \pi \times 10 \mathrm{kHz})=11.12723$, and $\widetilde{I}(\omega=$ $2 \pi \times 100 \mathrm{kHz})=11.1273$. This may be compared to the value $\lim _{R \rightarrow \infty}|D(R)|^{2}=D_{\text {at }}^{2}=\left|D_{2 \mathrm{~s}-2 \mathrm{~s}}+D_{2 \mathrm{~s}-2 \mathrm{p}}\right|^{2}=$ $\left|D_{2 \mathrm{~s}-2 \mathrm{p}}\right|^{2}=11.1272213$ obtained from the calculation described in Sec. III Clearly, the sum-rule (5) can also be used as a test for the correctness of numerical calculations. The very small deviations from the predicted sum-rule value may, however, not only be a result of an inaccuracy of the present numerical approach, but also reflect the (small) $R$ dependence of $D(R)$ that allows some $\omega$ dependence of the total photoassociation rate. This interpretation is supported by the fact that the numerically found deviations monotonously increase with increasing frequency $\omega$. Larger values of $\omega$ lead to a spatially more confined $\Psi^{10^{\prime}}(R ; \omega)$ which in turn probes more of the $R$-dependent part of $D(R)$. Since $D(R)$ approaches $D(R=\infty)=D_{\text {at }}$ from above, a small increase of $\widetilde{I}$ is expected for increasing trap frequencies. As is evident from Fig. 团(a) (especially the insert), the sumrule fulfillment is achieved by a drastic decrease of the photoassociation rate to the highest lying final states. This compensates the trap-induced increased rate to the lower lying states. Since the rate to the highest lying states is orders of magnitude larger than the one to the low-lying states, the reduced transition probability of a small number of states can easily compensate the substantial increase by orders of magnitude observed for the large number of low-lying states.

From Eq. (5) it is clear that in those cases where most of the contribution to the sum rule stems from the $R$ range where $D(R)$ is practically constant, there is also no influence of the initial-state wave function. Taking $D$ out of the integral yields always the self-overlap of the initial-state wave function and thus unity. This is important, since it indicates that the sum-rule value is also (approximately) independent of the atom-atom interaction potential.

\section{Enhancement and suppression factor $f^{v}$}

In order to quantify the effect of a tight harmonic trap on the photoassociation rate and to get rid of its variation as a function of the final-state vibrational level $v$ (that is due to the nodal structure and clearly visible in Fig.4(a) especially for smaller $v$ ) the ratio

$$
f^{v}(\omega)=\frac{I^{v}(\omega)}{I^{v}\left(\omega_{\mathrm{ref}}\right)} .
$$

may be introduced. It describes the relative enhancement $\left(f^{v}(\omega)>1\right)$ or suppression $\left(f^{v}(\omega)<1\right)$ of the photoassociation rate to a specific final state $v$ at a given trap frequency $\omega$ with respect to the reference frequency $\omega_{\text {ref }}$. Although it may appear to be most natural to choose the trap-free case as reference $\left(\omega_{\text {ref }}=0\right)$, a finite value offers some advantages. First, a different normalization applies to $\omega=0$ and $\omega \neq 0$, since in one case it is free-tobound transitions, while it is bound-to-bound transitions otherwise. Second, from a numerical point of view it is more convenient to treat both cases the same way and to avoid the variation of the box boundary $R_{\max }$ that would otherwise be necessary for the trap-free case. Finally, it may be argued that a non-zero trap reference state is in fact more relevant to typical photoassociation experiments with ultracold alkali atoms, since most of them are anyhow performed in traps. In the present work $\omega_{\text {ref }}=2 \pi \times 1 \mathrm{kHz}$ was chosen. This value is sufficiently small to represent typical shallow traps in which the influence of the trap on photoassociation is supposedly (at least to a good approximation) negligible. On the other hand, it allows to calculate the transition dipole moments with reasonable numerical efforts and thus sufficient accuracy.

The ratio $f^{v}(\omega)$ is shown for two different trap frequencies $\omega$ in Fig. U b). For most of the vibrational final 
states a simple constant regime is observed, i. e. the ratio $f^{v}(\omega)$ is independent of $v$ for all except the highest lying states. This constant regime is followed by a relatively sharp cut-off beyond which the ratio $f^{v}(\omega)$ is very small. In the constant regime a $100 \mathrm{kHz}$ trap leads to an enhancement by almost 3 orders of magnitude.

Comparing the results for different $\omega$ one notices that in the range of final states where a constant behavior (with respect to $v$ ) is observed, a tighter trap leads to an increased photoassociation rate, trap-induced enhanced photoassociation (EPA). Due to the cut-off this is, however, not true, if the last vibrational states are considered. Since the range of constant behavior shrinks with increasing trap frequency, there is an increasing range of vibrational states for which a tighter trap leads to a smaller photoassociation rate compared to a shallower trap. In this case trap-induced suppressed photoassociation (SPA) occurs. This effect is especially visible from the insert of Fig.4(a). The physical origin of the two different regimes (constant vs. cut-off) and their $\omega$ dependence is discussed separately in the following two subsections.

\section{Constant regime}

Since in the constant regime the ratio $f^{v}(\omega)$ is completely independent of the final state level $v$, its value (for a given $\omega$ ) and constancy (as a function of $v$ ) must be a consequence of the influence of the trap on the initial state. The initial-state wave function for a ${ }^{6} \mathrm{Li}$ atom pair was shown for three different trap frequencies in Fig. [3] $A$ view on the complete wave function reveals directly the confinement of the wave function to a smaller spatial volume, if the trap frequency is increased. However, on this scale the variation of the wave function at a specific value of $R$ appears to be quite complicated. Thus it is not at all clear why the enhancement factor $f^{v}$ has for so many states a constant value. A closer look at smaller internuclear separations (insert of Fig. 3) reveals that besides the initial oscillatory part confined to the effective range of the atom-atom interaction potential there is a relatively large $R$ interval in which the wave functions for the different trap frequencies vary linearly with $R$. In this case the slope is very small and the wave function is thus almost constant. If the Franck-Condon integral is determined only by the value of the initial-state wave function in this $R$ window, it produces an almost undistorted image of the final-state wave function.

However, for the ratio $f^{v}(\omega)$ this final-state dependence disappears. The reason is that in the $R$ range where the initial-state wave function is almost constant, its variation with the trap frequency is also $R$ independent, as can be seen from the insert of Fig. 3 In other words, one finds $\Psi^{10^{\prime}}(R ; \omega)=C(\omega) \cdot \Psi^{10^{\prime}}\left(R ; \omega_{\text {ref }}\right)$. If no final-state dependence occurs, the constant $C(\omega)$ is related to the ratio $f$ via $f_{c}(\omega)=C^{2}(\omega)$. The validity of this argument for the occurrence of a constant ratio $f$ can thus be checked (and visualized) in the following way. Together with the correct wave function $\Psi^{10^{\prime}}(R ; \omega)$ the approximate one, $\sqrt{f_{c}(\omega)} \cdot \Psi^{10^{\prime}}\left(R ; \omega_{\text {ref }}\right)$, is plotted where $f_{c}(\omega)$ is the value of the factor $f$ in the constant regime. A convenient way to determine $f_{c}(\omega)$ follows from the observation that the constant regime always starts at $v=0$. Thus $f_{c}(\omega)=f^{v=0}(\omega)$ is the most straightforward way of $f_{c}(\omega)$ determination. In Fig. [5 the correct wave function $\Psi^{10^{\prime}}(R ; \omega)$ is plotted together with the approximate wave function $\sqrt{f_{c}(\omega)} \cdot \Psi^{10^{\prime}}\left(R ; \omega_{\text {ref }}\right)$ for the trap frequency $\omega=2 \pi \times 100 \mathrm{kHz}$. The agreement between the

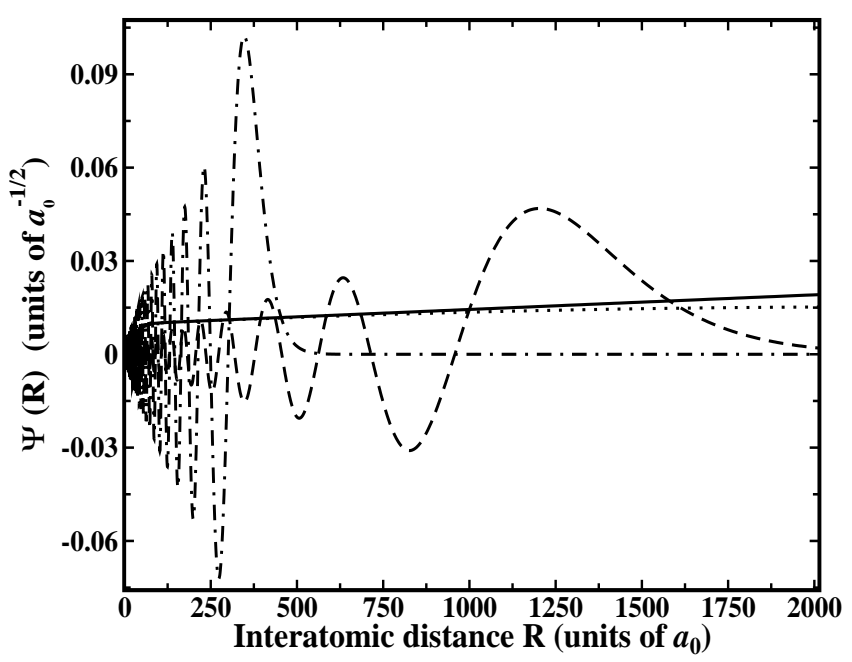

FIG. 5: Illustration of the constant photoassociation regime. The initial-state wave function $\Psi^{10^{\prime}}(R ; \omega=100)$ (dots) and an approximation to it, $\sqrt{f_{c}(\omega=100)} \cdot \Psi^{10^{\prime}}(R ; \omega=1)$ (solid), are shown together with the final-state vibrational wave functions for $v=88$ (chain) and $v=94$ (dashes).

two wave functions is clearly good in the shown range of $R$ values, but it is better for small $R$, since at about $R=500 a_{0}$ the two wave functions start to disagree. Below $R=500 a_{0}$ the two wave functions agree completely with each other, even in the very short $R$ range where they possess an oscillatory behaviour.

The key for understanding the occurrence of the constant regime is that a variation of the trap frequency modifies the spatial extent of the initial-state wave function, but leaves its norm and nodal structure preserved. As a consequence, the wave function changes qualitatively only in the large $R$ range, while in the short range only the amplitude varies (by factor $C(\omega)$ ). The reason for this behaviour is that at small $R$ the wave function is practically shielded from the trap potential by the dominant atom-atom interaction.

Fig. 5 shows also two final-state wave functions $(v=88$ and 94). According to Fig. 4(b) the transition to $v=88$ belongs still to the constant regime $\left(f^{88}(\omega) \approx f_{c}(\omega)\right)$, though to its very end. The transition to $v=94$ does on the other hand not belong to this regime, since for the considered trap frequency $f^{94}(\omega)<f_{c}(\omega)$. As is evident from Fig. [5] a constant ratio $f^{v}(\omega)$ is observed 
as long as the final-state wave function $v$ is completely confined within an $R$ range in which the approximation $\Psi^{10^{\prime}}(R ; \omega) \approx C(\omega) \cdot \Psi^{10^{\prime}}\left(R ; \omega_{\text {ref }}\right)$ is well fulfilled. This is (for $\omega=2 \pi \times 100 \mathrm{kHz}$ ) the case for $v=88$ for which the wave function is confined within $R<600 a_{0}$, but not for $v=94$ whose outermost lobe has its maximum at about $1350 a_{0}$. Since the $R$ range in which the initial-state wave function can be approximated in the here discussed fashion decreases with increasing trap frequency, the range of $v$ values for which $f^{v}(\omega) \approx f_{c}(\omega)$ is valid diminishes with increasing trap frequency.

The following rule of thumb is found to determine those vibrational levels $v$ for which the relation $f^{v}(\omega) \approx f_{c}(\omega)$ starts to break down. For trap frequencies $\omega_{1}$ and $\omega_{2}$ (with $\omega_{2}>\omega_{1}$ ) one may define a difference $\Delta$ that quantifies the deviation of $C \cdot \Psi^{10^{\prime}}\left(R ; \omega_{1}\right)$ and $\Psi^{10^{\prime}}\left(R ; \omega_{2}\right)$ as $\Delta(R)=C \cdot \Psi^{10^{\prime}}\left(R ; \omega_{1}\right)-\Psi^{10^{\prime}}\left(R ; \omega_{2}\right)$ where $C=\sqrt{f_{c}\left(\omega_{2}\right)}$. For example, in Fig. 5] the difference $\Delta(R)$ is the distance between the solid curve and the dotted one. The relation $f^{v}(\omega) \approx f_{c}(\omega)$ breaks down for those final states $v$ whose classical turning point lies beyond $R_{0} . R_{0}$ itself is determined by $\Delta\left(R>R_{0}\right) \gtrsim 10^{-3}$. In other words, if the last lobe of the final wave function overlaps substantially with a region where the deviation defined by $\Delta$ is larger than about $10^{-3}$, a clear deviation from the constant regime is to be expected.

\section{E. Cut-off regime}

Once the constant regime of the ratio $f^{v}$ (for a given trap frequency) is left, $f^{v}$ is steadily decreasing with $v$, as is apparent from Fig. 4(b). The photoassociation rate displays then a rather sharp cut-off behavior (see insert of Fig. 4(a)). The most loosely bound vibrational states of the final electronic state have in the trap-free case the largest rate but possess a very small one in very tight traps. For those high-lying states the wave functions have a very highly oscillatory behavior for short $R$ values and a large lobe close to the classical turning point. This outermost lobe determines the Franck-Condon integral, if the initial-state wave function is sufficiently smooth in this $R$ range. In Fig. 6] the initial-state wave function is shown together with the ones for $v=96$ and 98 (for $\omega=2 \pi \times 100 \mathrm{kHz}$ ).

It is evident from Fig. [6 that for $v=96$ the overlap of the initial wave function with the last lobe of the final state is very large. In fact, for this trap frequency the overlap reaches its maximum for $v=96$ and 97 (see Fig. 4(a)), despite the fact that the trap-induced relative enhancement factor $f^{v}(\omega)$ is small (Fig. 4(b)). In the case of $v=98$ the transition rate is not only clearly smaller than for $v=96$ or 97 , but it is also much smaller than the rate obtained for the same level at much lower trap frequencies (10 or $1 \mathrm{kHz}$ ). Clearly, one has $f^{v=98}(\omega)<1$ and thus for $\omega=2 \pi \times 100 \mathrm{kHz}$ the level $v=98$ represents an example for a trap-induced sup-

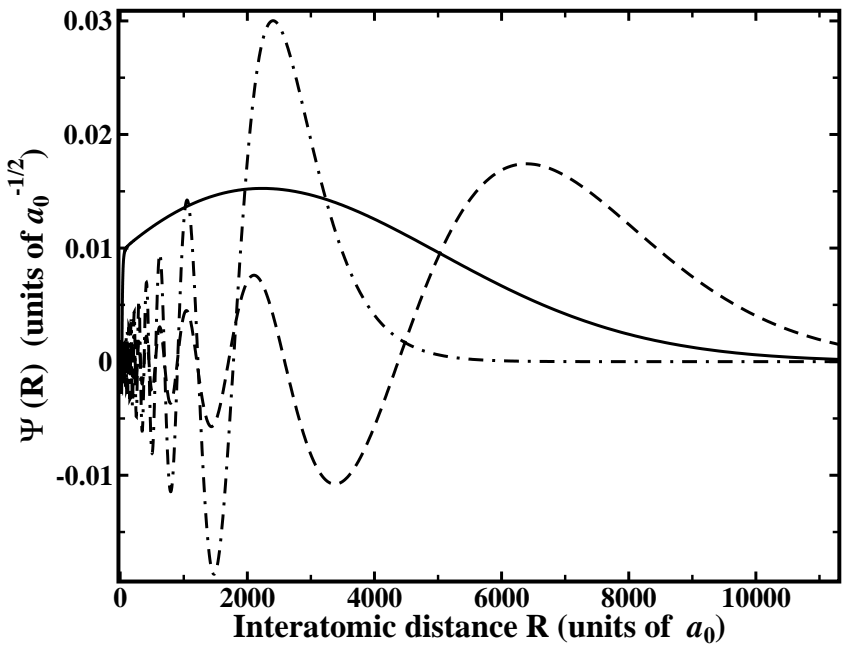

FIG. 6: Illustration of the cut-off regime for a $100 \mathrm{kHz}$ trap. The initial-state wave function $\Psi^{10^{\prime}}(R ; 100)$ (solid) is shown together with the two final-state wavefunctions for $v=96$ (chain) and $v=98$ (dashes).

pressed rate (SPA) in contrast to the usually expected enhanced photoassociation in a trap (EPA, $f^{v}(\omega)>1$ ). From Fig. [6 it is clear that the reason for the small transition rate to $v=98$ is due to the fact that the outermost lobe of the $v=98$ state lies mostly outside the $R$ range in which the initial-state wave function is non-zero. The least bound state (in the trap free case), $v=99$, possesses an even smaller photoassociation rate, since in this case the outermost lobe lies practically completely outside the non-zero $R$ range of the initial-state wave function. Due to the imperfect cancellation of the oscillating contributions from the inner lobes, the photoassociation rate for $v=99$ is very small, but non-zero.

Increasing the trap frequency even more will confine the initial-state wave function to a smaller $R$ range and thus SPA occurs for smaller $v$ values. The origin of the suppression is in fact a quite remarkable feature, since from Fig. 6] it is clear that the trap has practically no influence on the final states, even if one considers the highest-lying ones that have very tiny binding energies. This is still true, if the spatial extent of the final state is much larger than the one of the trap potential. This may be interpreted as a shielding of the trap potential by the molecular (atom-atom interaction) potential. The reason for the different shielding experienced by the initial and the final states is not only due to the fact that the former lies above the dissociation threshold, since then the photoassociation rate should dramatically increase, if transitions into the purely trap-induced bound states of the final electronic state are considered. This is, however, not the case as can be seen for the states $v>99$ in Fig. 4 (a). The different shielding is due to the inherently different long-range behaviors of the two electronic potential curves describing the initial $\left(a^{3} \Sigma_{u}^{+}\right)$and the final $\left(1^{3} \Sigma_{g}^{+}\right)$state. If one introduces the crossing point 
$R_{c}$ of the long range part of the van der Waals potential with the one of the inverted harmonic trap, it is defined by equating $C_{n} / R_{c}^{n}$ and $\frac{1}{2} \mu \omega^{2} R_{c}^{2}$ where $C_{n}$ is the corresponding leading van der Waals coefficient. At the point $R_{c}$ the trap potential starts to dominate. For example, in the case of the trap frequency $\omega=2 \pi \times 10 \mathrm{kHz}$ one finds $R_{c} \approx+825 a_{0}$ and $R_{c} \approx+17700 a_{0}$ for $a^{3} \Sigma_{u}^{+}$and $1^{3} \Sigma_{g}^{+}$of $\mathrm{Li}_{2}$, respectively.

\section{F. $I^{v}(\omega)$ for a repulsive interaction}

In order to check the main conclusions of the results obtained for ${ }^{6} \mathrm{Li}_{2}$ also the formation of ${ }^{39} \mathrm{~K}_{2}$ is investigated. While for ${ }^{6} \mathrm{Li}$ a photoassociation process between triplet states was considered, a transition between the $X^{1} \Sigma_{g}^{+}$and the $A^{1} \Sigma_{u}^{+}$states is chosen for ${ }^{39} \mathrm{~K}$. In contrast to the large negative scattering length of two ${ }^{6} \mathrm{Li}$ atoms interacting via the $a^{3} \Sigma_{u}^{+}$potential two ${ }^{39} \mathrm{~K}$ ground-state atoms interact via a small positive s-wave scattering length. The obtained results for the squared transition dipole moments $I^{v}(\omega)$ are qualitatively very similar to the results obtained for ${ }^{6} \mathrm{Li}_{2}$. This includes the existence of a constant regime of $f^{v}(\omega)$ followed by a pronounced decrease for the highest-lying vibrational states, the cut-off. The rule of thumb for predicting the range of $v$ values for which a constant ratio $f^{v}$ is observed does also work in this case. ${ }^{39} \mathrm{~K}_{2}$ shows thus trap-induced suppressed photoassociation for the highest lying states with a sharp cut-off in the $I^{v}(\omega)$ spectrum very much like ${ }^{6} \mathrm{Li}_{2}$. Therefore, the results are not explicitly shown for space reasons.

For a more systematic investigation of the influence of the scattering length $a_{\mathrm{sc}}$ and thus the type of interaction (sign of $a_{\mathrm{sc}}$ ) and its strength (absolute value of $a_{\mathrm{sc}}$ ) the mass of the $\mathrm{Li}$ atoms is varied. The mass variation allows for an in principle continuous (though non-physical) modification of $a_{\mathrm{sc}}$ from very large positive to negative values. With increasing mass an increasing number of bound states is supported by the same potential curve. Since $a_{\mathrm{sc}}$ is sensitive to the position of the least bound state, even a very small mass variation has a very large effect, if a formerly unbound state becomes bound. For example, an increase of the mass of ${ }^{6} \mathrm{Li}$ by $0.3 \%$ changes $a_{\mathrm{sc}}$ from $-2030 a_{0}$ to about $+850 a_{0}$. The (for ${ }^{6} \mathrm{Li}$ unbound) 11th vibrational state becomes weakly bound. A further increase of the mass increases its binding energy until it reaches the value for ${ }^{7} \mathrm{Li}$. It is also possible to modify $a_{\text {sc }}$ from $-2030 a_{0}$ to $+850 a_{0}$ by lowering the mass of ${ }^{6} \mathrm{Li}$. A larger mass variation is required (about $18 \%$ ) but the number of bound states remains unchanged. In this case the large positive value of $a_{\mathrm{sc}}$ indicates that the 10th bound state is, however, only very weakly bound and a further small decrease of the mass will shift it into the dissociative continuum.

In Fig. 17(a) $I^{v}(\omega)$ is shown for $a_{\mathrm{sc}}=+850 a_{0}$ (achieved by a $0.3 \%$ increase of the mass) and three different trap
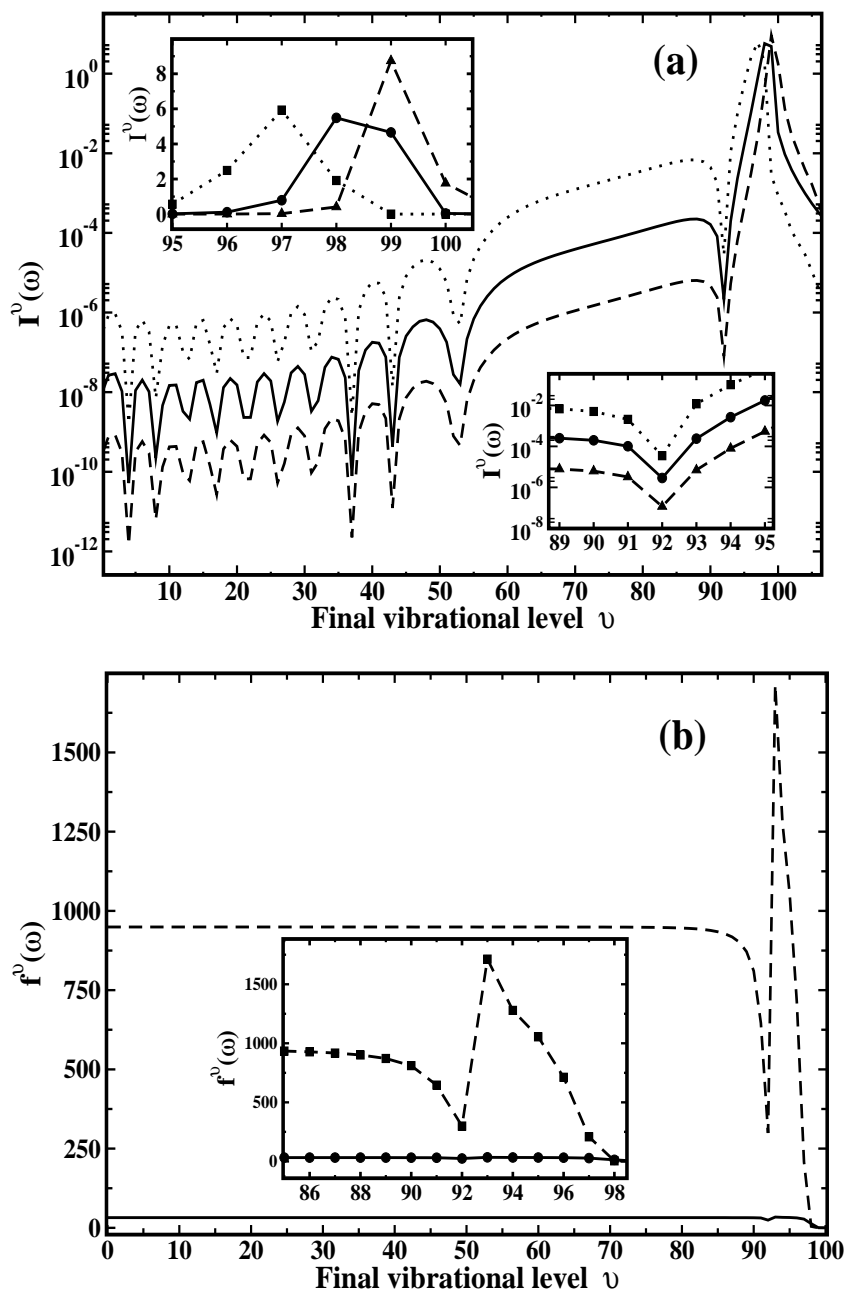

FIG. 7: As Fig. 4 but the scattering length is artificially changed to $a_{\mathrm{sc}}=+850 a_{0}$ (see text for details). The additional insert in the right bottom corner of (a) shows the range $v=89$ to $v=95$ on an enlarged scale.

frequencies as an example for a large positive scattering length and thus strong repulsive interaction. The overall result is again very similar to the one obtained for a large negative scattering length. A tighter trap increases the transition rate for most of the states, but there is a sharp cut-off for large $v$. The position of this cut-off moves to smaller $v$ as the trap frequency is increased. However, for a large positive value of $a_{\mathrm{sc}}$ an additional feature appears in the transition spectrum: a photoassociation window visible as a pronounced dip in the $I^{v}$ spectrum for large $v$. For the given choice of $a_{\mathrm{sc}}$ this minimum occurs for $v=92$.

The occurence of the dip for $a_{\mathrm{sc}} \gg 0$ has been predicted and explained for the trap-free case in 44, 45 and was experimentally confirmed [46]. Fig. 8 shows the last lobe of the final-state vibrational wave function $\Psi^{92}(R)$ together with the initial-state wave function, both for $\omega=2 \pi \times 100 \mathrm{kHz}$. The key for understanding the occurrence of the dip for large positive scattering lengths 


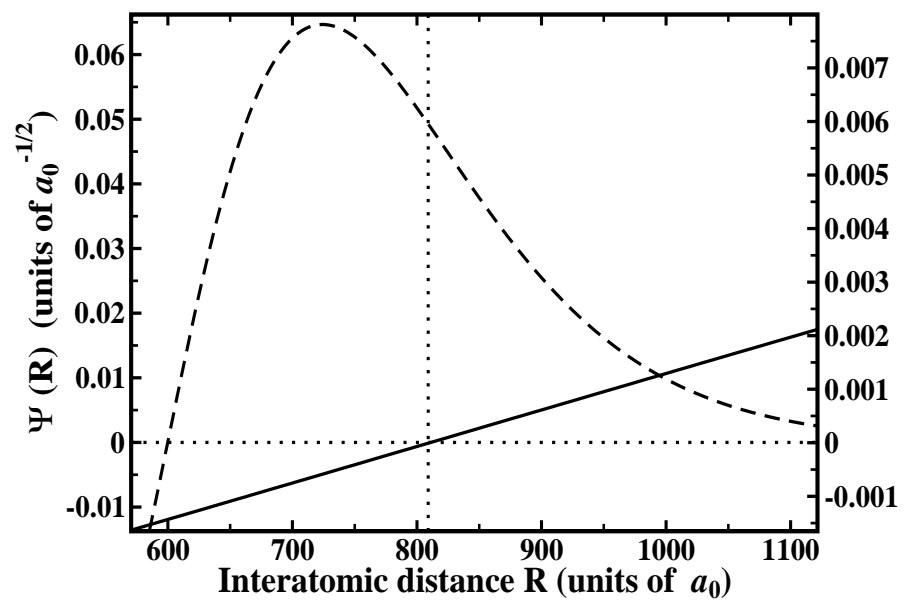

FIG. 8: For ${ }^{6} \mathrm{Li}_{2}$ (the scattering length in the initial channel is modified to $\left.a_{s c}=+850 a_{0}\right)$ and for a $100 \mathrm{kHz}$ trap the outermost lobe of the final-state wave function ( $v=92$, long dashes, left scale) is shown together with the initial-state wave function $\left(v^{\prime}=10\right.$, solid, right scale). Auxiliary horizontal and vertical dotted lines are given to assist the eye in identifying regions with a positive or negative overlap of the wavefunctions.

and its absence for negative ones is the change of sign of the initial-state wave function as a consequence of the repulsive atom-atom interaction. In fact, in the trap-free case the position of this node agrees of course with the scattering length. As can be seen from Fig. 8 the tight trap moves the nodal position to a smaller value, but this shift is comparatively small (about $5 \%$ ) even in the case of a $100 \mathrm{kHz}$ trap. For negative values of $a_{\mathrm{sc}}$ this node appears to be absent, since in this case only the extrapolated wave function intersects the $R$ axis, but this occurs at the non-physical interatomic separation $R_{\mathrm{x}}=-a_{\mathrm{sc}}$. As a result of the sign change occurring for $a_{\mathrm{sc}}>0$ the overlap of the initial-state wave function with a final state for which the mean position of the outermost lobe agrees with the nodal position $\left(R_{\mathrm{x}}\right)$ vanishes. The probability for a perfect agreement of those two positions is of course rather unlikely, but as can be seen from Fig. [7(a) and [4] where also an approximation for $I^{v}(\omega=0)$ was derived, the cancellation can be very efficient.

It should be emphasised that of course also for $a_{\mathrm{sc}}<0$ a number of dips occur as was discussed in the context of Fig.4 The difference between those dips and the one discussed for $a_{\mathrm{sc}} \gg 0$ is the occurrence of the latter outside the molecular regime. While the other dips are a direct consequence of the short-range part of the atom-atom interaction potential and thus confined (for $\mathrm{Li}_{2}$ ) to $v<55$ corresponding to $R<30 a_{0}$, the dip occuring for $a_{\mathrm{sc}} \gg 0$ can be located outside the molecular regime. This is even more apparent from Fig. 9] where the $I^{v}$ spectra for four different positive values of $a_{\mathrm{sc}}$ are shown together with the one for the (physical) value $a_{\mathrm{sc}}=-2030 a_{0}$ (all for $\omega=2 \pi \times 100 \mathrm{kHz})$. The values $a_{\mathrm{sc}}=+2020 a_{0},+350 a_{0}$, $+115 a_{0}$, and $+50 a_{0}$ were obtained by a mass increase of $\sim 0.3 \%, \sim 0.8 \%, \sim 2 \%$, and $\sim 6 \%$, respectively. In agree-

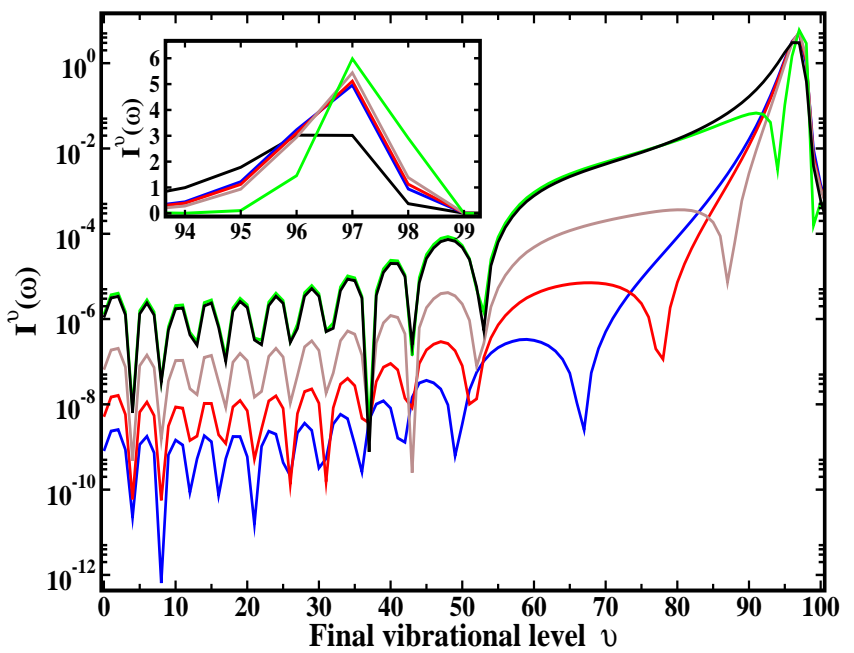

FIG. 9: (Color online) Dependence of the squared dipole transition moments $I^{v}(\omega)$ on the scattering length $a_{\mathrm{sc}}$ for transitions from the first trap-induced $\left(v^{\prime}=11\right)$ initial $a^{3} \Sigma_{u}^{+}$state to the vibrational manifold $(v)$ of the $1^{3} \Sigma_{g}^{+}$state of $\mathrm{Li}_{2}$ in a $\omega=2 \pi \times 100 \mathrm{kHz}$ trap. Using masses slightly larger than the one of ${ }^{6} \mathrm{Li} a_{s c}=+50 a_{0}$ (blue), $a_{s c}=+115 a_{0}$ (red), $a_{s c}=+350 a_{0}$ (brown), and $a_{s c}=$ $+2020 a_{0}$ (green) were yielded. For comparison, the result with the physical mass $\left(a_{s c}=-2030 a_{0}\right.$, black) is also shown. The insert shows the transitions to $v=94$ to 99 on an enlarged scale.

ment with the explanation given above, the position of the dip moves continuously to larger values of $v$ as the scattering length increases, since the position $R_{\mathrm{x}}$ of the last node of the initial state lies close to $a_{\mathrm{sc}}$. Also the positions of the other dips depend on $a_{\mathrm{sc}}$, but their dependence is much weaker and involves a much smaller $R$ interval. Clearly, the positions of the dips become more stable if they occur at smaller $v$.

Noteworthy, the positions of the first 10 dips agree perfectly for $a_{\mathrm{sc}}=-2030$ and $+2020 a_{0}$. In fact, both spectra are on a first glance in almost perfect overall agreement, except the occurrence of the additional dip for $v=92$. According to the discussion of the sum rule in Sec.IIIB the total sum $\tilde{I}$ should be (approximately) independent of the atomic interaction and thus $a_{\mathrm{sc}}$. This is also confirmed numerically for the present examples. The insert of Fig.9 reveals how the sum-rule is fulfilled. The due to the additional dip missing transition probability is compensated by an enhanced rate to the neighbor states with larger $v$.

In all shown cases with $a_{\mathrm{sc}}>0$ there exist 11 bound states in contrast to the 10 states of ${ }^{6} \mathrm{Li}\left(a_{\mathrm{sc}}=-2030 a_{0}\right)$. As mentioned in the beginning of this section, it is also possible to change the sign of $a_{\mathrm{sc}}$ while preserving the number of nodes. The corresponding $I^{v}$ spectra (again for $\omega=2 \pi \times 100 \mathrm{kHz}$ ) are shown in Fig.10. The same values of $a_{\mathrm{sc}}$ as in Fig.9] $\left(+2020 a_{0},+350 a_{0},+115 a_{0}\right.$, and $\left.+50 a_{0}\right)$ are now obtained by a decrease of the mass by $\sim 18 \%, \sim 17.5 \%, \sim 16 \%$, and $\sim 13 \%$, respectively. A comparison of the two Figs.9] and 10 demonstrates that the position of the outermost $\operatorname{dip}\left(\right.$ for $a_{\mathrm{sc}} \gg 0$ ) depends 


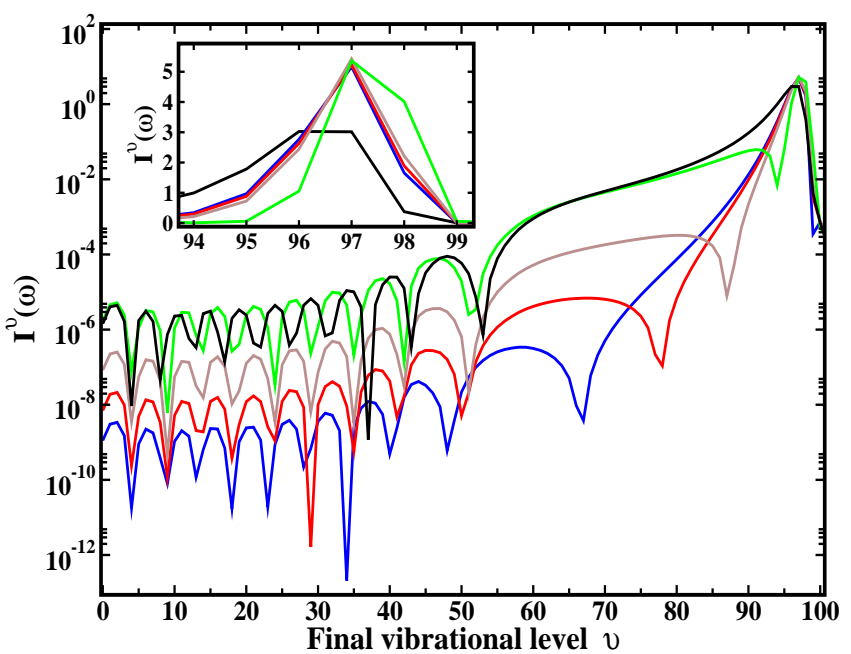

FIG. 10: (Color online) As Fig. 9 but for a variation of $a_{\mathrm{sc}}$ by means of a reduction of the mass with respect to the one of ${ }^{6} \mathrm{Li}$. In this case, the number of bound states remains unchanged and the transition starts from the $v^{\prime}=10 a^{3} \Sigma_{u}^{+}$state.

for a given $\omega$ solely on $a_{\mathrm{sc}}$, while the other dips (in the molecular regime) differ when changing the total number of bound states from 10 to 11 . A comparison of the results obtained for $a_{\mathrm{sc}}=-2030 a_{0}$ and $+2020 a_{0}$ with 10 bound states in both cases shows that most of the nodes in the molecular regime are shifted with respect to each other in such a way that the $v$ range hosting 10 dips for $a_{\mathrm{sc}}=-2030 a_{0}$ contains 9 dips for $a_{\mathrm{sc}}=-2030 a_{0}$.

Turning back to Fig. [7 and the question of the influence of a tight trap on the photoassociation rate for $a_{\mathrm{sc}} \gg 0$ one notices that the position of the additional dip appears to be practically independent of $\omega$. As was explained in the context of Fig. [8 the reason is that the position of the outermost node depends only weakly on $\omega$. For the shown example this shift is even for a $100 \mathrm{kHz}$ trap small compared to the separation of the outermost lobes between neighboring $v$ states. Therefore, the shift is not sufficient to move the dip position away from $v=92$. However, if $a_{\text {sc }}$ is, e. g. increased to $+2020 a_{0}$ the crossing point $R_{\mathrm{x}}$ shifts in a $100 \mathrm{kHz}$ trap to about $1500 a_{0}$ and changes thus by $\approx 25 \%$. In this case the dip position moves from $v=95$ to 94 . It is therefore important to take the effects of a tight trap into account, if they are used for the determination of $a_{\mathrm{sc}}$ using photoassociation spectroscopy the way discussed in [45, 46].

In order to focus on the effect of the tight trap it is again of interest to consider the ratio $f^{v}(\omega)$ introduced in Sec.IIIC For small but positive values of $a_{\mathrm{sc}}$ the ratio $f^{v}$ is structurally very similar to the case $a_{\mathrm{sc}}=-2030 a_{0}$ shown in Fig. 目(b). A uniform constant regime covering almost all $v$ states is followed by a sharp cut-off whose position shifts to smaller $v$ as $\omega$ increases. A similar behavior is encountered for $a_{\mathrm{sc}}=+850 a_{0}$ and $\omega=10 \mathrm{kHz}$ as shown in Fig.7(b). However, for a tighter trap $(100 \mathrm{kHz})$ a new feature appears. In this case the relative enhance- ment at the dip position $(v=92)$ is smaller than in the constant regime, but larger for the neighbor states. The enhancement factor for $v=92$ is only $\approx 25 \%$ of $f_{c}$, while the one for $v=93$ is $\approx 60 \%$ larger than $f_{c}$. This results in a dispersion-like structure in $f^{v}$. It should be emphasised that this is again remarkably different from the other dips in $I^{v}(\omega)(v<55)$ that show the same (constant) enhancement factor $f_{c}$ as their neighbor states.

\section{G. Combined influence of trap and atomic interaction}

In view of the very important question how the efficiency of photoassociation can be improved, Fig. 9] reveals that besides the use of a tight trap a large scattering length is also favorable. The photoassociation rate (away from the dips) is enhanced by orders of magnitude, if $a_{\mathrm{sc}}$ varies from $a_{\mathrm{sc}}=+50 a_{0}$ to $a_{\mathrm{sc}}=+2020 a_{0}$ ! In view of the already discussed fact that the results for the overall spectrum $I^{v}$ differ for $a_{\mathrm{sc}}>0$ and $a_{\mathrm{sc}}<0$ only by the position of the dips, it is evident that photoassociation (or corresponding Raman transitions) are much more efficient, if $\left|a_{\mathrm{sc}}\right|$ is very large.

In order to understand the dependence of the FC factors of the vibrational final states on the scattering length it is instructive to look at the variation of the initial-state wave function with $a_{\mathrm{sc}}$ for large $R$ values. This is shown in Fig. 11] for $\omega=2 \pi \times 100 \mathrm{kHz}$. While a large attrac-

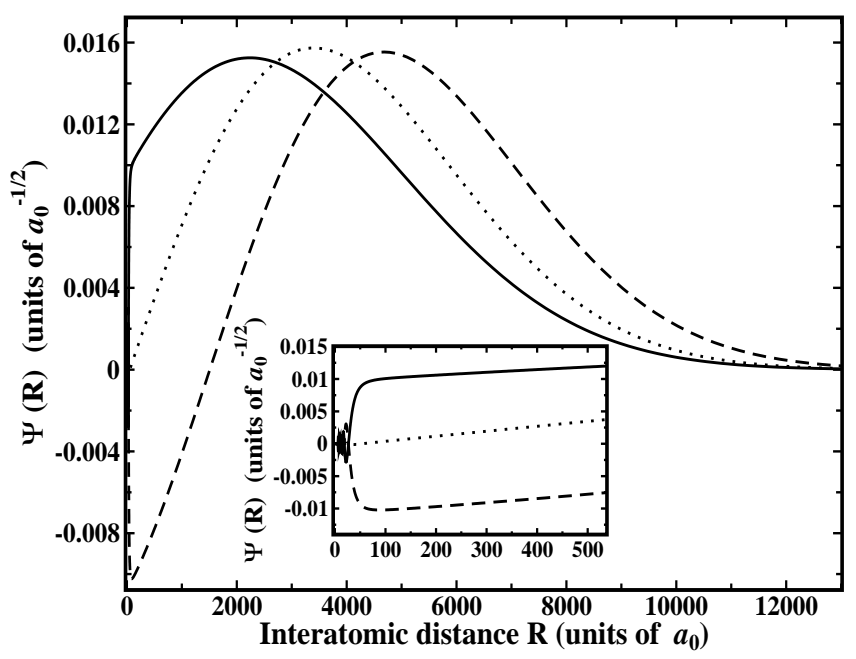

FIG. 11: Wave functions of the initial state describing two $\mathrm{Li}$ atoms in a trap with frequency $\omega=2 \pi \times 100 \mathrm{kHz}$ for different masses that yield the scattering lengths $a_{\mathrm{sc}}=-2030 a_{0}$ (solid), $a_{\mathrm{sc}}=+50 a_{0}$ (dots), and $a_{\mathrm{sc}}=+2020 a_{0}$ (dashes). The insert shows the small $R$ range on an enlarged scale.

tive interaction $\left(a_{\mathrm{sc}} \ll 0\right)$ leads to a very confined wave function for the first trap-induced bound state, a large repulsive interaction $\left(a_{\mathrm{sc}} \gg 0\right)$ does not only result in a node (responsible for the photoassociation window discussed above), but also to a push of the outermost lobe 
to larger $R$ values. This push is of course counteracted by the confinement of the trap. However, only the highest lying final states probe the very large $R$ range. As is apparent from Fig. 2 the final states $v \leq 92$ probe almost completely the range $R \leq 1000 a_{0}$. Within this $R$ interval the absolute value of the initial-state wave function increases with the absolute value of $a_{\mathrm{sc}}$. As a consequence, the corresponding $\mathrm{FC}$ factors and $I^{v}$ should increase with $\left|a_{\mathrm{Sc}}\right|$. An exception to this is the already discussed occurrence of the photoassociation window (spectral dip) that occurs for a positive scattering length, if the position of the node is probed by the final-state wave function. Consequently, one expects for the low-lying final states (in fact for almost all except the very high-lying ones and the ones at the dip position) that an increase of $\left|a_{\mathrm{sc}}\right|$ leads to an increased photoassociation rate.

An evident question is of course, whether the enhancements due to the use of tighter traps and tuning of $a_{\mathrm{sc}}$ can be used in a constructive fashion? In order to investigate this question, one can introduce another enhancement factor

$$
g^{v}\left(\omega, a_{\mathrm{sc}}\right)=\frac{I^{v}\left(\omega, a_{\mathrm{sc}}\right)}{I^{v}\left(\omega_{\mathrm{ref}}, a_{\mathrm{sc}, \mathrm{ref}}\right)}
$$

with $a_{\text {sc,ref }}=0 a_{0}$ (and $\omega_{\text {ref }}=2 \pi \times 1 \mathrm{kHz}$ as before). Clearly, a cut through $g^{v}\left(\omega, a_{\mathrm{sc}}\right)$ for constant $a_{\mathrm{sc}}$ is equal to $f^{v}(\omega)$. A cut for constant $\omega$ describes on the other hand the relative enhancement of the photoassociation rate as a function of $a_{\mathrm{sc}}$.

The function $g^{v}\left(\omega, a_{\mathrm{sc}}\right)$ depends of course on the vibrational state $v$, but as was discussed before, most of the states show a constant enhancement factor $f_{c}$. Thus it is most interestingly to investigate $g_{c}\left(\omega, a_{\mathrm{sc}}\right)$ that is defined as the $g$ function for vibrational states for which the relation $f^{v}=f_{c}$ is valid. This excludes the states in the cut-off regime and those at or very close to the photoassociation window. In Fig. $12 g_{c}\left(\omega, a_{\mathrm{sc}}\right)$ is shown as a function of $a_{\mathrm{sc}}$ for different trap frequencies. The important finding is that $g_{c}\left(\omega, a_{\mathrm{sc}}\right)$ increases as a function of $\omega$ and $\left|a_{\mathrm{sc}}\right|$. In fact, within the shown ranges of $\omega$ and $a_{\mathrm{sc}}$ the function $g_{c}\left(\omega, a_{\mathrm{sc}}\right)$ raises by 6 orders of magnitude, if the maximum values of $\omega(2 \pi \times 100 \mathrm{kHz})$ and $a_{\mathrm{sc}}\left( \pm 2000 a_{0}\right)$ are considered! A more detailed analysis shows that the enhancement is almost equally distributed among the two parameters, i. e. a factor $10^{3}$ stems from the variation of $\omega$ and about the same factor from varying $a_{\mathrm{sc}}$. Thus the enhancement of the photoassociation rate due to the two different physical parameters occurs practically independently of each other, at least in the rather large parameter range considered. It should be emphasized that these ranges are realistically achievable in present-day experiments. It is interesting to note that this finding is not only very encouraging with respect to the possible enhancement of photoassociation rates and related molecule production schemes, but it shows also that the influence of the parameters scattering $\left(a_{\mathrm{sc}}\right)$ and characteristic length scale of an isotropic harmonic trap $\left(a_{\mathrm{ho}}=\sqrt{1 /(\mu \omega)}\right)$ on the photoassociation process

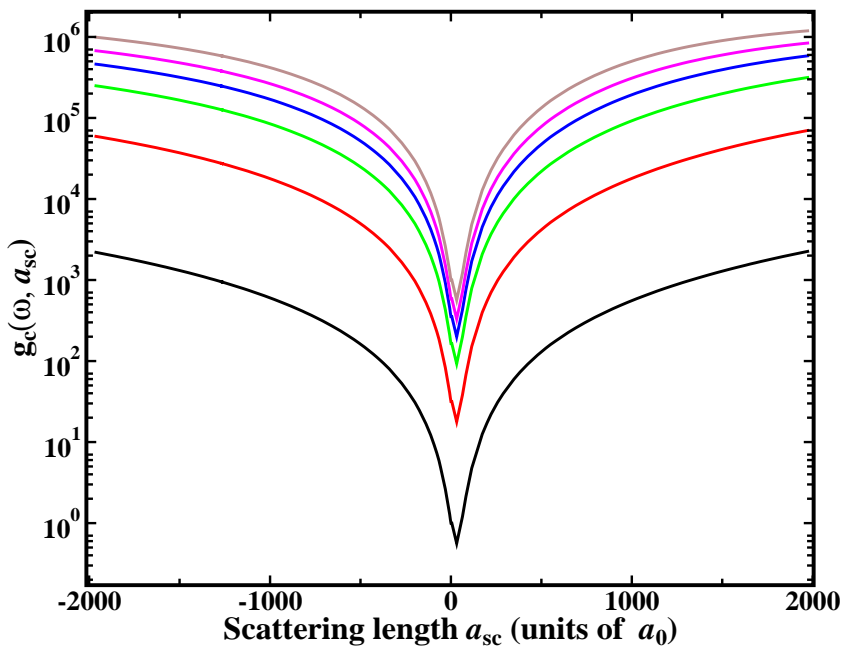

FIG. 12: (Color online) Enhancement factor $g_{c}$ [see Eq. 7]] in the constant ( $v$-independent) regime as a function of $a_{\text {sc }}$ for trap frequencies $\omega=2 \pi \times 1 \mathrm{kHz}$ (black), $\omega=2 \pi \times 10 \mathrm{kHz}$ (red), $\omega=$ $2 \pi \times 30 \mathrm{kHz}$ (green), $\omega=2 \pi \times 50 \mathrm{kHz}$ (blue), $\omega=2 \pi \times 70 \mathrm{kHz}$ (purple), and $\omega=2 \pi \times 100 \mathrm{kHz}$ (brown).

is very different from the one observed for the energy. In energy-related discussions (like the one on the validity of the pseudopotential approximation in 20]) it was found that the ratio $\left|a_{\mathrm{sc}} / a_{\mathrm{ho}}\right|$ determines the behavior. In the present case, both parameters and not only their ratio are important.

\section{PSEUDOPOTENTIAL APPROXIMATION}

The bound state of two atoms in a harmonic trap when the atom-atom interaction $V_{\mathrm{int}}(R)$ is approximated by a regularized contact potential $\frac{4 \pi}{2 \mu} a_{\mathrm{sc}} \delta^{3}(\vec{R}) \frac{\partial}{\partial R} R$ with energy-independent scattering length $a_{\mathrm{sc}}$, was first derived analytically by Busch at al. [18]. The bound states with integer quantum number $n_{t}$ are expressed as

$$
\Psi_{a_{\mathrm{sc}}}^{n_{t}}(R)=\frac{1}{2} \pi^{-3 / 2} A R e^{-\bar{R}^{2} / 2} \Gamma(-\nu) U\left(-\nu, \frac{3}{2}, \bar{R}^{2}\right)
$$

with $\bar{R}=R / a_{\text {ho }}$ and the characteristic length scale $a_{\text {ho }}$ of the harmonic trap introduced in the end of the previous section. $A$ is a normalization constant having the dimension of the inverse of the square root of volume (see below) and $\nu$ is an effective quantum number for the relative motional eigenstate, $\nu=\frac{E_{a_{\mathrm{sc}}}^{n_{t}}}{2 \omega}-\frac{3}{4}$. The energy eigenvalues are given by the roots of the equation

$$
\frac{\Gamma(-x / 2+3 / 4)}{\Gamma(-x / 2+1 / 4)}=\frac{1}{\sqrt{2} \xi},
$$

where $x=E_{a_{\mathrm{sc}}}^{n_{t}} / \omega$ and $\xi=a_{\mathrm{sc}} / a_{\mathrm{ho}}$.

The initial-state wave function $\Psi^{10^{\prime}}(R ; \omega)$ of two ${ }^{6} \mathrm{Li}$ atoms interacting through the $a^{3} \Sigma_{u}^{+}$potential and the 
pseudopotential wave function $\Psi_{a_{\mathrm{sc}}}^{0}$ with the physical (trap-free) value of the scattering length $a_{\mathrm{sc}}=-2030 a_{0}$ are plotted together in Fig. 13 for the case of a trap frequency $\omega=2 \pi \times 10 \mathrm{kHz}$. As expected, wave function

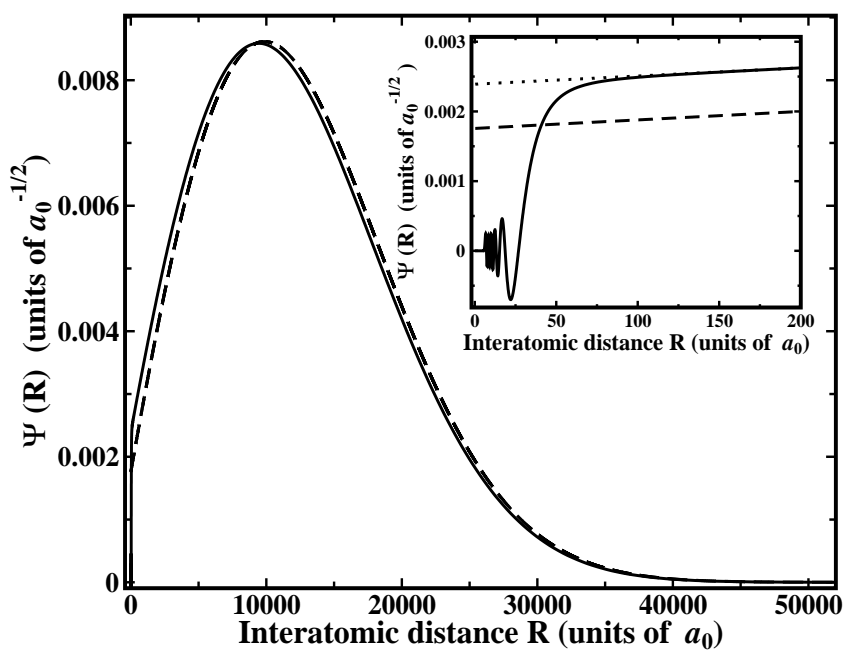

FIG. 13: Wave functions of the first trap-induced bound state $(\omega=$ $2 \pi \times 10 \mathrm{kHz})$ of two ${ }^{6} \mathrm{Li}$ atoms interacting through the full $a^{3} \Sigma_{u}^{+}$ potential (solid), a pseudopotential with the energy-independent (trap-free) scattering length $a_{\mathrm{sc}}=-2030 a_{0}$ (dashes), and one with the energy-dependent value $a_{E}=-2872 a_{0}$ (dots). The insert shows the short $R$ range on an enlarged scale.

$\Psi_{a_{\mathrm{sc}}}^{0}$ fails completely for short internuclear separations, since it does not reproduce any nodal structure at all. In addition, $\Psi_{a_{\mathrm{sc}}}^{0}$ possesses a wrong behavior at $R=0$ where it is non-zero. In the long-range part $\Psi_{a_{\mathrm{sc}}}^{0}$ agrees better with the correct wave function. There the main difference is an evident phase shift between the two functions. This phase shift is a consequence of the trap and vanishes in the absence of the trap $(\omega \rightarrow 0)$. The physical reason for the phase shift is the non-zero ground-state energy in a trap (zero-point energy and motion) due to the Heisenberg uncertainty principle. As a consequence, the scattering of the two atoms in a trap differs from the trap-free case even at zero temperature. On the basis of an analysis of the energy spectrum of two atoms in a harmonic trap it was found that a pseudopotential approximation using an energy-dependent scattering length $a_{E}$ leads to a highly improved description of two particles confined in an isotropic harmonic trap [20, 47].

While the scattering length is defined in the limit $E \rightarrow 0$, an energy-dependent scattering length can be introduced by extending its original asymptotic definition in terms of the phase shift for s-wave scattering $\delta_{0}(E)$ to non-zero collision energies. This yields $a_{E}=$ $-\tan \delta_{0}(E) / k$ with $k=\sqrt{2 \mu E}$. Clearly, the evaluation of $\delta_{0}(E)$ requires to solve the complete scattering problem and thus also $a_{E}$ can only be obtained from the knowledge of the solution for the correct atom-atom interaction potential.

The values of $a_{E}$ were obtained in the following way.
After a determination of the ground-state energy of two ${ }^{6} \mathrm{Li}$ atoms from a full calculation (using the realistic interaction potential), this energy is used in Eq. (9) to find $a_{E}$ (that is inserted in the equation in place of $a_{\mathrm{sc}}$ ). More details about this so-called self-consistency approach are given in [48]. In this way an energy-dependent scattering length $a_{E}=-2872 a_{0}$ is, e.g., found for two ${ }^{6} \mathrm{Li}$ atoms in a trap with frequency $\omega=2 \pi \times 10 \mathrm{kHz}$. The resulting wave function is also shown in Fig. 113 together with the correct one and the one obtained for $a_{\mathrm{sc}}=-2030 a_{0}$. Clearly, the agreement with the correct wave function is very good for large $R$. For $R>150 a_{0}$ the wave function obtained for $a_{E}=-2872 a_{0}$ is not distinguishable from the correct one. Only in the insert of Fig. 13 that shows the wave functions at short internuclear separations one sees a deviation. It is caused by the absence of any nodal structure and the wrong behavior at $R \rightarrow 0$ of the pseudopotential wave function. In fact, at short distances the introduction of an energy-dependent scattering length that corrects the phase shift leads to an even larger error compared to the use of $a_{\mathrm{sc}}$.

The validity of the pseudopotential approximation using an energy-dependent scattering length has been discussed before. In 20] it was found that applicability of this approximation depends on the ratios $\beta_{6} / a_{\mathrm{ho}}$ and $\left|a_{\mathrm{sc}} / a_{\mathrm{ho}}\right|$ where $\beta_{6}=\left(2 \mu C_{6}\right)^{1 / 4}$ is the characteristic length scale of the interaction potential in the case of a leading $C_{6} / R^{6}$ van der Waals potential. For two ${ }^{6} \mathrm{Li}$ atoms in a trap with $\omega=2 \pi \times 100 \mathrm{kHz}$ that interact via the $a^{3} \Sigma_{u}^{+}$potential those ratios are 0.02 and 0.59 , respectively. These validity criteria are, however, based solely on energy arguments. In other words, if those ratios are sufficiently smaller than 1 , the energy obtained by means of Eq. (9) with $a_{\mathrm{sc}}$ should agree well with the correct one. In the present example of ${ }^{6} \mathrm{Li}$ the ratio between the correct first trap-induced energy $E^{10^{\prime}}$ and $E_{a_{\mathrm{sc}}}^{n_{t}=0}$ obtained with the energy-independent pseudopotential is $E^{10^{\prime}} / E_{a_{\mathrm{sc}}}^{0}=0.96$ for $\omega=2 \pi \times 10 \mathrm{kHz}$ and $E^{10^{\prime}} / E_{a_{\mathrm{sc}}}^{0}=0.92$ for $\omega=2 \pi \times 100 \mathrm{kHz}$. By construction, the energy $E_{a_{\mathrm{E}}}^{n_{t} 0}$ agrees of course completely with $E^{10^{\prime}}$.

In Fig. 14] $I^{v}(\omega)$ obtained when using the pseudopotential approximation with energy-independent scattering length is compared to the spectrum obtained for the correct atom-atom interaction, both for a trap frequency $\omega=2 \pi \times 10 \mathrm{kHz}$. The two results disagree completely for $v \leq 60$. For higher lying vibrational states $(v>60)$ the agreement is reasonable. (Note, however, the logarithmic scale.) For the highest lying states $(v \geq 95)$ very good agreement is found even on a linear scale (see insert of Fig. 14). Adopting the energy-dependent scattering length yields quantitative agreement already for $v \geq 75$, but again a complete disagreement for $v \leq 60$.

The breakdown of the pseudopotential approximation (with energy-independent or dependent scattering length) for describing photoassociation to the low-lying vibrational states is, of course, a direct consequence of the wrong short-range behavior of the pseudopotential 


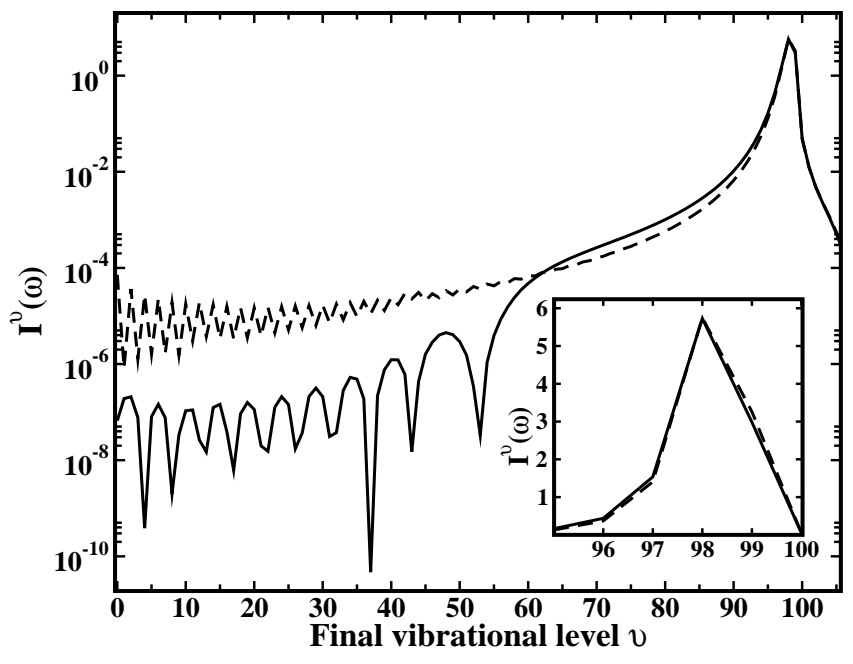

FIG. 14: Squared photoassociation transition moments $I^{v}(\omega)$ for ${ }^{6} \mathrm{Li}$ in a $\omega=2 \pi \times 10 \mathrm{kHz}$ trap calculated with the molecular interaction potential (solid) or within the energy-independent pseudopotential approximation (dashes).

wave functions (Fig. 113). From the definition of $I^{v}(\omega)$ it follows that the pseudopotential approximation fails, if the final-state vibrational wave function has a substantial amplitude in the $R$ range in which the initial-state wave function is strongly influenced by the atom-atom interaction. An estimate for this $R$ range is (in the present case) the already discussed effective-range parameter $\beta_{6}=\left(2 \mu C_{6}\right)^{1 / 4}$. Since for large $v$ the final-state wave function is dominated by its outermost lobe whose position is in turn close to the classical outer turning point $R_{\text {out }}$, the pseudopotential approximation should be valid for $R_{\text {out }}>\beta_{6}$. In the case of ${ }^{6} \mathrm{Li}$ one finds $\beta_{6}=62.5 a_{0}$. According to Fig. 22 the pseudopotential approximation should thus be applicable for $v>70$. A recently performed photoassociation experiment for ${ }^{6} \mathrm{Li}$ considered the transition to $v=59$ [12]. For this specific example the pseudopotential approximation would predict a two times smaller rate than the full calculation for $\omega=2 \pi \times 10 \mathrm{kHz}$.

The validity of the pseudopotential approximation for predicting the photoassociation rates to the high lying states can also be used to investigate whether the simulation of different scattering lengths by mass scaling is senseful. This could be questionable, since a change of the mass does not only modify the scattering length (by moving the position of the least bound state), but also the kinetic energy term. Thus it may be argued that the discussed influence of the scattering length on the photoassociation process could partly also be a consequence of the modification of the kinetic energy, at least in the case of a substantial mass variation as it was required for preserving the number of bound states. Within the pseudopotential approximation the scattering length is, however, a parameter independent of the mass. Therefore, in contrast to the case of the full calculation it is possible within the pseudopotential approximation to investigate the isolated influence of a variation of the scattering length (keeping the mass fixed). A corresponding analysis confirms that mass scaling can in fact be used to simulate a modified atom-atom interaction.

The pseudopotential approximation was used already in 21] for an analysis of the change of the photoassociation rate due to a scattering-length modification. The investigation concentrated, however, on very high lying vibrational states close to or even above the trap-free dissociation limit. Since for transitions to those states the $R$ dependence of the electronic transition dipole moment can safely be ignored, it is sufficient to concentrate on the Franck-Condon (FC) factors. In Fig. 15 the squares of these factors are shown as a function of the scattering length for $90 \leq v \leq 98$ and trap frequency $\omega=2 \pi \times 100 \mathrm{kHz}$. As in 21] the pseudopotential approximation is used for the initial state, but here the final-state wave function is obtained by a full numerical calculation while an approach based on quantum defect theory (QDT) was used in 21]. Furthermore, $\mathrm{Na}_{2}$ was considered in 21] while it is $\mathrm{Li}_{2}$ in the present study.

For the states $90 \leq v \leq 93$ shown in Fig. 15 (a) the dependence on $a_{\mathrm{sc}}$ in a $100 \mathrm{kHz}$ trap is very similar to the one found in 21]. The rather regular variation with $a_{\mathrm{sc}}$ is due to the fact that the final-state wave function probes the flat part of the initial-state wave function, as can be seen in the insert of Fig. 15(a) where the wave function for $v=92$ is shown together with the initialstate wave function for three different values of $a_{\mathrm{sc}}$. The initial-state wave function varies almost linearly with $a_{\mathrm{sc}}$ in the Franck-Condon window of the $v=92$ final state. According to the discussion in Sec.IIIE for a $100 \mathrm{kHz}$ trap the states $v \geq 90$ belong to the cut-off regime, but for $v \leq 93$ the enhancement factor $f^{v}$ is still close to its value $f_{c}$ in the constant regime (see Figs. 4 and 7). The minima of the $\mathrm{FC}^{2}$ factors for $a_{\mathrm{sc}} \gg 0$ are a consequence of the dip discussed in Sec.IIIF since the nodal position $R_{\mathrm{x}}$ moves to larger $R$ if $a_{\mathrm{sc}}$ increases, the minimum in the $\mathrm{FC}^{2}$ factors moves to a larger value of $a_{\mathrm{sc}}$ if $v$ increases. While the pseudopotential approximation is capable to predict the existence of the dip occuring for $a_{\mathrm{sc}} \gg 0$, its position is not necessarily correctly reproduced in a trap. This is due to the fact that the pseudopotential overestimates the trap-induced shift of the position of the outermost node. For example, if the mass of $\mathrm{Li}$ is varied such that $a_{\mathrm{sc}}=+850 a_{0}$ is obtained, a $100 \mathrm{kHz}$ trap shifts $R_{\mathrm{x}}$ to $\approx+810 a_{0}$ (Fig. [8) and the dip occurs at $v=92$ (Fig. [7). Using the pseudopotential approximation (with $\left.a_{\mathrm{sc}}=+850 a_{0}\right)$ yields on the other hand $R_{\mathrm{x}} \approx+580 a_{0}$ and the dip occurs for $v=90$. This error in the prediction of $R_{\mathrm{x}}$ increases with $a_{\mathrm{sc}}$.

The final states $94 \leq v \leq 98$ whose $\mathrm{FC}^{2}$ factors are shown in Fig. 15 (b) probe on the other hand the nonlinear part of the initial-state wave function (close to the trap boundary). Consequently, the dependence on $a_{\mathrm{sc}}$ differs from the one found in 21]. While for $90 \leq v \leq 92$ the $\mathrm{FC}^{2}$ factors are first decreasing and then increasing, 

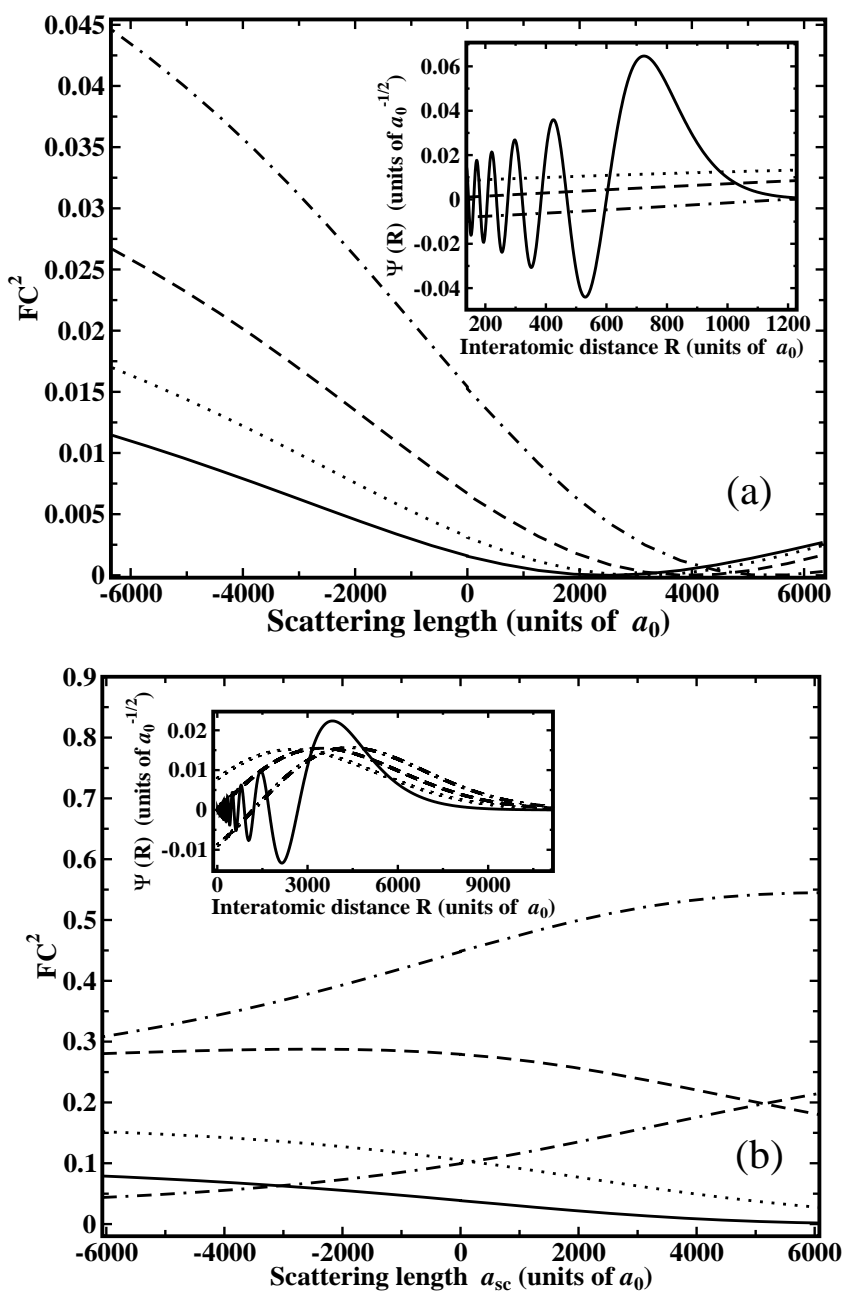

FIG. 15: (a) Squared Franck-Condon factors between the final vibrational states $v=90$ (solid), 91 (dots), 92 (dashes), or 93 (chain) of the $1^{3} \Sigma_{g}^{+}$state and the initial-state pseudopotential wave functions as a function of the scattering length $a_{\mathrm{sc}}$. The trap frequency is $\omega=2 \pi \times 100 \mathrm{kHz}$. The insert shows the $v=92$ finalstate wave function together with the pseudopotential wavefunctions for $a_{s c}=-2000 a_{0}$ (dots), $a_{s c}=0$ (dashes), $a_{s c}=+2000 a_{0}$ (chain). (b) As (a), but for $v=94$ (solid), 95 (dots), 96 (dashes), 97 (chain), $\mathrm{v}=98$ (dot-dash-dash). The insert shows the $v=97$ final-state wavefunction and the pseudopotential wave functions for $a_{s c}=-2000 a_{0}$ (dots), $a_{s c}=0$ (dashes), $a_{s c}=+2000 a_{0}$ (chain).

if $a_{\text {sc }}$ varies from $-6000 a_{0}$ to $+6000 a_{0}$, the ones of $93 \leq$ $v \leq 96$ are purely decreasing. For $v=97$ and 98 the $\mathrm{FC}^{2}$ factors are on the other hand increasing with $a_{\mathrm{sc}}$.

In view of the fact that the scattering length of a given atom pair may be known (for example from some measurement), but the corresponding atom-atom interaction potential is unknown, it is of course interesting to investigate whether the pseudopotential approximation allows to predict the enhancement factor also in the constant regime, i. e. whether it correctly reproduces $f_{c}(\omega)$. This would allow for a simple estimate of the effect of a tight trap on the photoassociation rate in the constant regime that covers most of the spectrum. In order to determine $f_{c}(\omega)$ it is sufficient to analyze the ratio of the initial-state wave function $\Psi_{a_{\mathrm{sc}}}^{0}$ for the trap frequencies $\omega$ and $\omega_{\text {ref }}$. This comparison may be done at any arbitrary internuclear separation $R_{\text {lin }}$ provided it belongs to the linear regime. The result is

$$
f_{c}^{\text {pseudo }}(\omega)=\left[\frac{\Psi_{a_{\mathrm{sc}}}^{0}\left(R_{\mathrm{lin}} ; \omega\right)}{\Psi_{a_{\mathrm{sc}}}^{0}\left(R_{\mathrm{lin}} ; \omega_{\mathrm{ref}}\right)}\right]^{2} .
$$

A very special and simple choice that guarantees that $R_{\text {lin }}$ belongs to the linear regime is $R_{\text {lin }}=0$. With this value of $R_{\text {lin }}$ one finds from the analysis of $\Psi_{a_{\mathrm{sc}}}^{0}$

$$
f_{c}^{\text {pseudo }}(\omega)=\left[\frac{A(\omega)}{A\left(\omega_{\mathrm{ref}}\right)}\right]^{2} \frac{\omega_{\mathrm{ref}}}{\omega},
$$

where $A(\omega)$ is the normalization factor fulfilling $|A(\omega)|^{2}=\sqrt{2 \omega} \pi \xi^{2} \frac{\partial E}{\partial \xi}[18]$. Depending on the level of approximation one may use either $a_{\mathrm{sc}}$ or $a_{E}$ in the evaluation of $A$. An even simpler estimate of the influence of a tight harmonic trap on the photoassociation rate is obtained, if the atom-atom interaction potential is completely ignored in the initial state. The harmonicoscillator eigenfunctions at $R=R_{\text {lin }}=0$ yield

$$
f_{c}^{\text {ho }}(\omega)=\left(\frac{\omega}{\omega_{\text {ref }}}\right)^{3 / 2}
$$

In Fig. [16 the enhancement factors $f_{c}(\omega)$ calculated at the different levels of approximation are shown as a function of the trap frequency $\omega$. The results obtained for ${ }^{6} \mathrm{Li}$

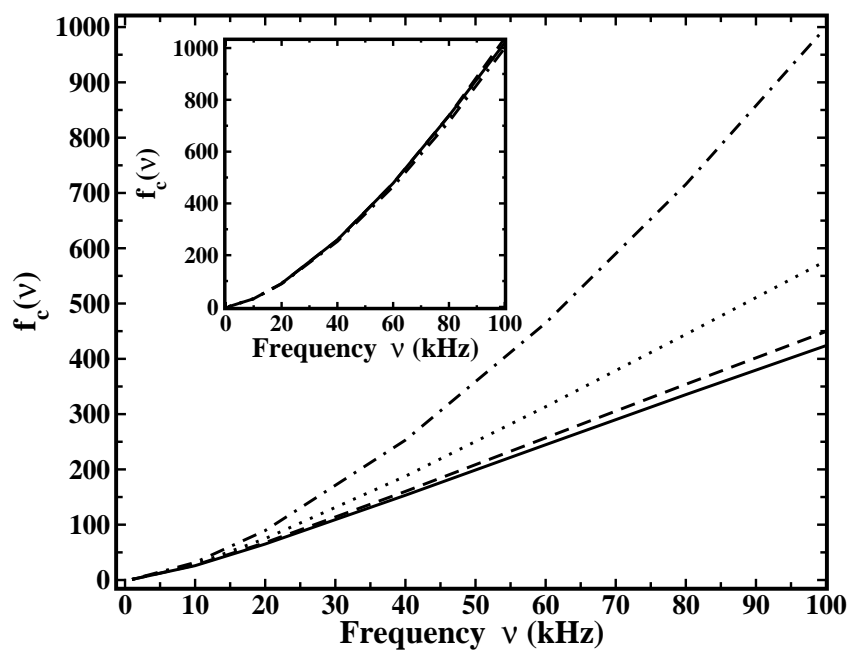

FIG. 16: Dependence of the enhancement factor $f_{c}$ on the trap frequency $\nu=\omega /(2 \pi)$ for ${ }^{6} \mathrm{Li}_{2}$ using the molecular (solid), the energy-independent (dots) and -dependent (dashes) pseudopotential, or the harmonic-oscillator (chain) wave functions. The insert shows the same curves but for ${ }^{39} \mathrm{~K}_{2}$.

and ${ }^{39} \mathrm{~K}$ are compared to each other. Remind, in the latter case the scattering length $a_{\mathrm{sc}}=+90 a_{0}$ has a much smaller absolute value than for ${ }^{6} \mathrm{Li}\left(a_{\mathrm{sc}}=-2030 a_{0}\right)$. 
Consequently, one expects the atom-atom interaction to be less important. This is confirmed by (the insert of) Fig. 16] The results obtained for $f_{c}(\omega)$ with the aid of the different approximations discussed above are in very good agreement with the correct result in the case of ${ }^{39} \mathrm{~K}$. Even the simple harmonic-oscillator model predicts the enhancement factor in the constant regime very accurately.

It should be emphasized that the correct prediction of the enhancement factor by the simplified approximation works, although the prediction of the rates is completely wrong (Fig.14) in this constant regime (small $v$ ). In the case of a large absolute value of the scattering length (like for ${ }^{6} \mathrm{Li}$ ), i.e. for a strong atom-atom interaction, the frequency dependence of $f_{c}(\omega)$ predicted by the simplified models is on the other hand not very accurate. In fact, the simple harmonic-oscillator model clearly overestimates the enhancement factor for large $\omega$. The pseudopotential approximation yields much better results, especially if the energy-dependent scattering length $a_{E}$ is used. (As already mentioned, $a_{E}$ is, however, only available from the knowledge of the exact atom-atom interaction.)

In view of the usefulness of Eq. (11) for obtaining an estimate of the enhancement factor $f_{c}(\omega)$ but the rather complicated procedure to calculate $\frac{\partial E}{\partial \xi}$ required for obtaining $A(\omega)$, it is interesting to test whether $A(\omega)$ can alternatively be evaluated from an expansion of the energy $E$ at $\xi=0$. Using the relation $\frac{\partial x}{\partial \xi}=\left(\frac{\partial \xi}{\partial x}\right)^{-1}$ it is straightforward to determine with the aid of (9) an expansion for the scaled energy

$$
x(\xi)=\frac{3}{2}+\left.\sum_{n=0}^{\infty} \frac{1}{(n+1) !} \frac{\partial^{(n)} F(x)}{\partial x^{(n)}}\right|_{x=3 / 2} \xi^{n+1}
$$

with

$$
F(x)=-\frac{2 \sqrt{2} \Gamma\left[\frac{3}{4}-\frac{x}{2}\right]}{\Gamma\left[\frac{1}{4}-\frac{x}{2}\right] \psi\left[\frac{1}{4}-\frac{x}{2}\right]-\psi\left[\frac{3}{4}-\frac{x}{2}\right]}
$$

and the digamma function $\psi$. The zero- and first-order terms of the expansion (13) are given in [18]. Using Eqs. (13) and (11)

$$
f_{c}^{\text {pseudo }}(\omega)=\frac{\left.\sum_{n=0}^{\infty} \frac{1}{n !} \frac{\partial^{(n)} F(x)}{\partial x^{(n)}}\right|_{x=3 / 2} \xi^{n}}{\left.\sum_{n=0}^{\infty} \frac{1}{n !} \frac{\partial^{(n)} F(x)}{\partial x^{(n)}}\right|_{x=3 / 2} \xi_{\text {ref }}^{n}}\left(\frac{\omega}{\omega_{\text {ref }}}\right)^{\frac{3}{2}}
$$

is obtained with $\xi_{\text {ref }}=\frac{a_{\mathrm{sc}}}{a_{\mathrm{ho}, \mathrm{ref}}}$. In Fig. 17 the 4 th-, 5th, and 6th-order expansions are compared to the results obtained with the non-approximated term (all for $a_{\mathrm{sc}}=$ $\left.-2030 a_{0}\right)$ and with the correct atom-atom interaction result. Note, Eq. (15) can also be used for the evaluation of $g_{c}\left(\omega, a_{\mathrm{sc}}\right)$, if in the denominator $\xi_{\text {ref }}$ is replaced by $\tilde{\xi}_{\text {ref }}=\frac{a_{\mathrm{sc}, \mathrm{ref}}}{a_{\mathrm{ho}, \mathrm{ref}}}$.

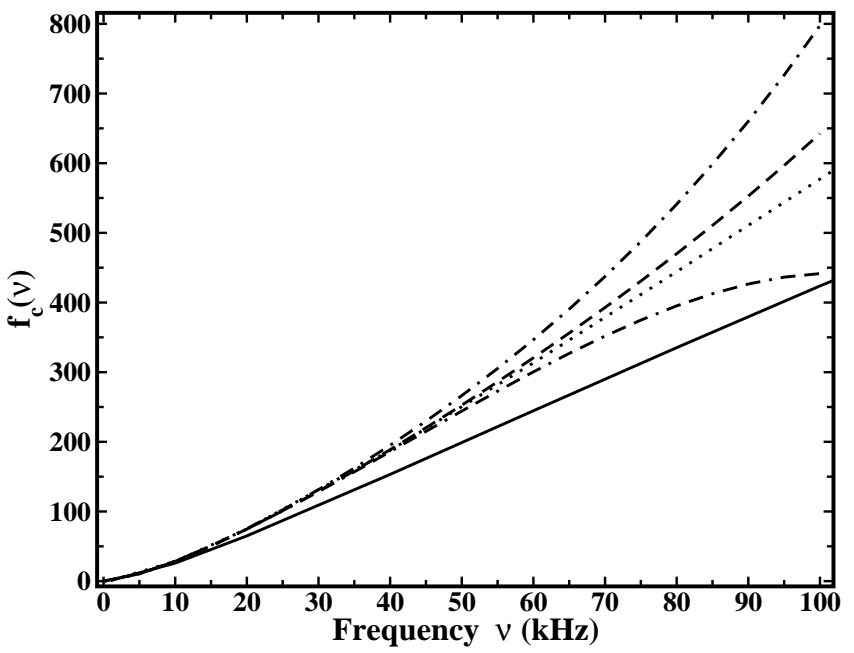

FIG. 17: Investigation of the convergence of the series expansion 115 for the enhancement factor $f_{\mathrm{C}}^{\text {pseudo }}(\omega)$ in the constant regime. Shown are the results of the full (infinite order, dotted) and the 4th (dashes), 5th (chain), and 6th (dot-dash-dash) order expansion. For comparison, also the enhancement factor obtained with the molecular potential is shown (solid).

\section{DISCUSSION AND OUTLOOK}

In this work the influence of a tight isotropic harmonic trap on the photoassociation process has been investigated for alkali atoms. It is found that for most of the states (the ones in the constant regime) there is an identical enhancement as the trap frequency increases. This enhancement can reach 3 orders of magnitude for trap frequencies of about $100 \mathrm{kHz}$ as they are reported in literature. While the enhancement itself agrees at least qualitatively with the concept of confinement of the initialstate wave function, also trap-induced suppressed photoassociation is possible. In fact, as a simple sum rule confirms, any enhancement must be accompanied by suppression. The physical origin of this suppression is the trap-induced confinement of the initial-state wave function of relative motion within a radius that is smaller than the mean internuclear separation of the least bound vibrational states in the electronic target state. Since in the present calculation both initial and final state are exposed to the same harmonic trap, this result may appear surprising. While the explanation is based on the different long-range behaviors of the two involved electronic states, the effect itself may be very interesting in terms of, e.g., quantum information.

Consider for example an optical lattice as trapping potential. The initial (unbound) atom pair is (for sufficient trap depths) located within a single lattice site (Mott insulator state). In the photoassociated state it could, however, reach into and thus communicate with the neighbor site, if the lattice parameters are appropriately chosen. Such a scenario could be used for a controlled logical operation (two-qubit gate) like the CNOT. Since the latter 
forms together with single-qubit gates a universal gate, this could provide a starting point for a quantum computer. Alternatively, it may be interesting to use the fact that if a single spot with the dimension of the trap length $a_{\text {ho }}$ or a specific site in an optical lattice can be addressed, then the atoms would only respond, if they are in their (unbound) initial state. If they are in the photoassociated excited state, they would on the other hand be located outside the trap and thus would not respond. For this it is already sufficient, if they are (predominantly) located in the classically forbidden regime. Also, modifying the trap frequency it is possible to block the photoassociation process on demand. The trap frequency is then varied in such a fashion that a specific final state resonantly addressed with a laser with sufficiently small bandwidth belongs either to the constant or to the cut-off regime.

A further important finding of this work is that the influence of a tight trap on the photoassociation spectra (as a function of the final vibrational state) for different alkali atoms is structurally very similar, independent whether photoassociation starts from the singlet or triplet ground state. Also the type of interaction (strong or weak as well as repulsive or attractive) does not lead to a substantial modification of the trap influence. The only exception is a strong repulsive interaction that leads to a pronounced window in the photoassociation spectrum. The reason is the position of the last node in the initial-state wave function that in this case is located at a relatively large value of $R$ and leads to a cancellation effect in the overlap with the final state. The nodal position depends strongly on $a_{\mathrm{sc}}$, but only for very tight traps also on $\omega$. As has been discussed previously [44, 46], the position of the window may be used for a scattering-length determination. This will also approximately work for not too tight traps, but the trap influence has to be considered for very tight ones. Alternatively, the window provides a control facility, since the transition to a single state can be selectively suppressed. In very tight traps this effect is not only more pronounced, but in addition the transitions to the neighbor states are further enhanced. This could open up a new road to control in the context of the presently on-going discussion of using femtosecond lasers for creating non-stationary wave packets in the electronic excited state [49, 50, 51]. One of the problems encountered in this approach is the difficulty to shape the wave packet, since the high-lying vibrational states that have a reasonable transition rate are energetically very closely spaced and thus the shape of the wavepacket is determined by the Franck-Condon factors that cannot easily be manipulated but strongly increase as a function of $v$.

In view of the question how to enhance photoassociation or related association schemes (like Ramanbased ones) the investigation of the enhancement factors $g^{v}\left(\omega, a_{\mathrm{sc}}\right)$, especially its value in the constant regime $\left(g_{c}\left(\omega, a_{\mathrm{sc}}\right)\right)$ is most important. It shows that not only increasing the tightness of the trap (enlarging $\omega$ ) leads to an enhancement of the photoassociation rate, but a sim- ilar effect can be achieved by increasing the interaction strength $\left|a_{\mathrm{sc}}\right|$. Most interestingly, these two enhancement factors work practically independent of each other, i. e. it is possible to use both effects in a constructive fashion and to obtain a multiplicative overall enhancement factor. For a $100 \mathrm{kHz}$ trap and a scattering length $\left|a_{\mathrm{sc}}\right|$ of the order of 2000 an enhancement factor (uniform for all states in the constant regime) of 5 to 6 orders of magnitude is found compared to the case of a shallow $1 \mathrm{kHz}$ trap and $\left|a_{\mathrm{sc}}\right|=0$.

A comparison of the results obtained for the correct atom-atom interaction potential with the ones obtained using the approximate pseudopotential approximation or ignoring the interaction at all shows that these approximations yield only for the transitions to very high lying vibrational states a good estimate of the photoassociation rate. Nevertheless, despite the complete failure of predicting the rates to low lying states, these models allow to determine the enhancement factor in the constant regime. For weakly interacting atoms (small $\left|a_{\mathrm{sc}}\right|$ ) already the pure harmonic-oscillator model (ignoring the atomic interaction) leads to a reasonable prediction of the trap-induced enhancement factor $f_{c}(\omega)$.

It is important to stress that the results in this work were obtained for isotropic harmonic traps with the same trapping potential seen by both atoms. In this case center-of-mass and relative motion can be separated and in both coordinates an isotropic harmonic trap potential (with different trap lengths due to the different total and reduced masses) is encountered. As is discussed, e.g., in 19, 47] where a numerical and an analytical solution are respectively derived for the case of atoms interacting via a pseudopotential, a similar separation of center-ofmass and relative motion is possible for axially symmetric (cigar or pancake shaped) harmonic traps.

In reality, the traps for alkali atoms are of course not strictly harmonic. Since the present work focuses, however, for the initial atom pair on the lowest trap induced state the harmonic approximation should in most cases be well justified. Independently on the exact way the trap is formed (for example by a far off-resonant focused Gaussian laser beam or by an optical lattice), the lowest trapinduced state agrees usually well with the one obtained in the harmonic approximation, if the zero-point energy is sufficiently small. This requirement sets of course an upper scale to the applicability of the harmonic approximation with respect to the trap frequency. If $\omega$ is too large, the atom pair sees the anharmonic part of the trap. (Clearly, the trap potential must also be sufficiently deep to support trap-induced bound states, i.e. to allow for Mott insulator states in the case of an optical lattice).

An additional problem arises from the anharmonicity of a real trap: the anharmonic terms lead to a nonseparability of the relative and the center-of-mass motion. In fact, a recent work discusses the possibility of using this coupling of the two motions for the creation of molecules [14]. Again, a tighter trap is expected to lead to a stronger coupling and thus finally to a breakdown of 
the applicability of the harmonic model.

For the final state of the considered photoassociation process there exists on the first glance an even more severe complication. Usually, the two atoms will not feel the same trapping potential, since they populate different electronic states. In the case of traps whose action is related to the induced dipole moment (which is the case for optical potentials generated with the aid of lasers that are detuned from an atomic transition), the two atoms (in the case of $\mathrm{Li}$ the ones in the $2^{2} \mathrm{~S}$ and the $2^{2} \mathrm{P}$ state) will in fact see potentials with opposite sign. If the laser traps the ground-state atoms, it repels the excited ones. In the alternative case of an extremely far-off resonant trap the trapping potential is proportional to the dynamic polarizability of the atoms. In the long-wavelength limit (as is realized, e.g., in focused $\mathrm{CO}_{2}$ lasers 52]) the dynamic polarizability approaches the static one, $\lim _{\lambda \rightarrow \infty} \alpha(\lambda)=\alpha_{\text {st }}$. The static polarizabilities do not necessarily have opposite signs for the ground and the excited electronic state of an alkali atom, but in many cases different values. Then the trapping potentials for the initial and final states of the photoassociation process are different. The Li system appears to be a counter example, since for ${ }^{6} \mathrm{Li}_{2}$ the average polarizability of the $a^{3} \Sigma_{u}^{+}(2 \mathrm{~s}+2 \mathrm{~s})$ state is predicted to be equal to $\bar{\alpha}=\alpha_{z z}=\alpha_{x x}=2 \alpha_{0}(2 s)=2 \times 165=330 a_{0}$ For the $1^{3} \Sigma_{g}^{+}\left(2 \mathrm{~s}+2 p_{z}\right)$ state one has $\alpha_{z z}=\alpha_{0}(2 s)+\alpha_{z z}\left(2 p_{z}\right)=$ $285 a_{0}$ and $\alpha_{x x}=\alpha_{0}(2 s)+\alpha_{x x}\left(2 p_{z}\right)=292 a_{0}$ yielding an average polarizability $\bar{\alpha} \approx 290 a_{0}[53$. Thus the trapping potentials are expected to be very similar. This is not the case for, e.g., ${ }^{87} \mathrm{Rb}_{2}$ where the average polarization for the $a^{3} \Sigma_{u}^{+}$state is $670 a_{0}$ and for $1^{3} \Sigma_{g}^{+}$it is $1698 a_{0}[\underline{54}$.

It was checked that the use of very different values of $\omega$ for determining the initial and final state wave functions does not influence the basic findings of the present work. The reason is simple. Besides the very least bound states (and of course the trap-induced ones) the final states are effectively protected by the long-range interatomic potential from seeing the trap. However, if the two atoms are exposed to different trap potentials, a separation of center-of-mass and relative motion is again not possible, even in the fully harmonic case (a fact that was, e.g., overlooked in 15]). One would again expect that this coupling increases with the difference in the trap potentials of the two involved states. A detailed study of the consequences of the coupling of center-of-mass and relative motion due to various reasons like an anharmonicity of the trap or different trapping potentials seen by the involved atoms is presently underway. This involves also the case of the formation of heteronuclear alkali dimers where besides the occurrence of this coupling for the initial state also a different long-range behavior of the interatomic potential has to be considered.

Different interaction strengths occur naturally for dif- ferent alkali atoms as is well known and also evident from the explicit examples of ${ }^{6} \mathrm{Li},{ }^{7} \mathrm{Li}$, and ${ }^{39} \mathrm{~K}$ that were discussed in this work. According to the findings of this work the choice of a proper atom pair (with large $\left|a_{\mathrm{sc}}\right|$ ) enhances the achievable photoassociation yield quite dramatically. Clearly, for practical reasons it is usually not easy to change in an existing experiment the atomic species, since the trap and cooling lasers are adapted to a specific one. In addition, the naturally existing alkali species provide only a fixed and limited number of interaction strengths.

The tunability of the interaction strength on the basis of Feshbach resonances, especially magnetic ones, marked a very important corner-stone in the research area of ultracold atomic gases. The findings of the present work strongly suggest that this tunability could be used to improve the efficiency of photoassociation (and related) schemes. However, it has to be emphasized that it is not at all self-evident that the independence of the scattering-length variation and the one of the trap frequency as it occurs in the model used in this work is applicable to (magnetic) Feshbach resonances. Furthermore, the present work considered only the single-channel case while the proper description of a magnetic Feshbach resonance requires a multi-channel treatment. Noteworthy, a strong enhancement of the photoassociation rate by at least 2 orders of magnitude while scanning over a magnetic Feshbach resonance was predicted on the basis of a multichannel calculation for a specific ${ }^{85} \mathrm{Rb}$ resonance already in 55]. An experimental confirmation followed very shortly thereafter [56]. The explanation for the enhancement given in [55] is, however, based on an increased admixture of bound-state contribution to the initial continuum state in the vicinity of the resonance. This is evidently different from the reason for the enhancement due to large values of $\left|a_{\text {sc }}\right|$ discussed in the present work.

An extension within the multichannel formalism that allows for a full treatment of magnetic Feshbach resonances is presently underway.

\section{Acknowledgments}

The authors acknowledge financial support by the Deutsche Forschungsgemeinschaft within the SPP 1116 (DFG-Sa936/1) and SFB 450. AS is grateful to the Stifterverband für die Deutsche Wissenschaft (Programme Forschungsdozenturen) and the Fonds der Chemischen Industrie for financial support. This work is also supported by the European COST Programme D26/0002/02.
[1] M. H. Anderson, J. R. Ensher, M. R. Matthews, C. E. Wieman, and E. A. Cornell, Science 269, 198 (1995).
[2] S. Jochim, M. Bartenstein, A. Altmeyer, G. Hendl, 
S. Riedl, C. Chin, J. Hecker Denschlag, R. Grimm, C. Zhu, A. Dalgarno, et al., Science 302, 2101 (2003).

[3] C. A. Regal, C. Ticknor, J. L. Bohn, and D. S. Jin, Nature 424, 3 (2003).

[4] M. W. Zwierlein, C. A. Stan, C. H. Schunck, S. M. F. Raupach, S. Gupta, Z. Hadzibabic, and W. Ketterle, Phys. Rev. Lett. 91, 250401 (2003).

[5] P. D. Lett, K. Helmerson, W. D. Phillips, L. P. Ratliff, S. L. Rolston, and M. E. Wagshul, Phys. Rev. Lett. 71, 2200 (1993).

[6] A. Fioretti, D. Comparat, A. Crubellier, O. Dulieu, F. Masnou-Seeuws, and P.Pillet, Phys. Rev. Lett. 80, 4402 (1998)

[7] P. D. Drummond, K. V. Kheruntsyan, D. J. Heinzen, and R. H. Wynar, Phys. Rev. A 65, 063619 (2002).

[8] E. R. I. Abraham, W. I. McAlexander, C. A. Sackett, and R. G. Hulet, Phys. Rev. A 74, 1315 (1995).

[9] E. Tiemann, H. Knckel, and H. Richling, Eur. Phys. J. D 37, 323 (1996).

[10] A. Fioretti, C. Amiot, C. Dion, O. Dulieu, M. Mazzoni, G. Smirne, and C. Gabbanini, Eur. Phys. J. D 15, 189 (2001).

[11] R. Gutterres, C. Amiot, A. Fioretti, C. Gabbanini, M. Mazzoni, and O. Dulieu, Phys. Rev. A 66, 024502 (2002).

[12] U. Schloeder, T. Deuschle, C. Silber, and C. Zimmermann, Phys. Rev. A 68, 051403 (2003).

[13] D. Jaksch, V. Venturi, J. I. Cirac, C. J. Williams, and P. Zoller, Phys. Rev. Lett. 89, 040402 (2002).

[14] E. L. Bolda, E. Tiesinga, and P. S. Julienne, Phys. Rev. A 71, 033404 (2005).

[15] C. P. Koch, F. Masnou-Seeuws, and R. Kosloff, Phys. Rev. Lett. 94, 193001 (2005).

[16] M. Greiner, O. Mandel, T. Esslinger, T. Hänsch, and I. Bloch, Nature 415, 39 (2002).

[17] T. Rom, T. Best, O. Mandel, A. Widera, M. Greiner, T. W. Hänsch, and I. Bloch, Phys. Rev. Lett. 93, 073002 (2004).

[18] T. Busch, B.-G. Englert, K. Rzazewski, and M. Wilkens, Found. of Phys. 28, 549 (1998).

[19] Z. Idziaszek and T. Calarco, Phys. Rev. A 71, 050701 (2005).

[20] D. Blume and C. H. Greene, Phys. Rev. A 65, 043613 (2002).

[21] B. Deb and L. You, Phys. Rev. A 68, 033408 (2003).

[22] M.-L. Almazor, O. Dulieu, M. Elbs, E. Tiemann, and F. Masnou-Seeuws, Eur. Phys. J. D 5, 237 (1999).

[23] M.-L. Almazor, O. Dulieu, F. Masnou-Seeuws, R. Beuc, and G. Pichler, Eur. Phys. J. D 15, 355 (2001).

[24] M. Kemmann, I. Mistrik, S. Nussmann, and H. Helm, Phys. Rev. A 69, 022715 (2004).

[25] F. D. Colavecchia, J. P. Burke, W. J. Stevens, M. R. Salazar, G. A. Parker, and R. T. Pack, J. Chem. Phys. 118, 5484 (2003).

[26] C. Linton, T. Murphy, F. Martin, R. Bacis, and J. Verges, J. Chem. Phys. 91, 6036 (1989).

[27] I. Schmidt-Mink, W. Müller, and W. Meyer, Chem. Phys. 92, 263 (1985).

[28] M. Marinescu and A. Dalgarno, Phys. Rev. A 52, 311 (1995).
[29] Y. V. Vanne, private communication.

[30] Y. V. Vanne and A. Saenz, J. Phys. B: At. Mol. Phys. 37, 4101 (2004).

[31] S. Magnier, M. Aubert-Frécon, J. Hanssen, and C. L. Sech, J. Phys. B: At. Mol. Phys. 32, 5639 (1999).

[32] I. Dumitriu, Y. V. Vanne, and A. Saenz (2006), to be published.

[33] L. B. Ratcliff, J. L. Fish, and D. D. Konowalow, J. Mol. Spec. 122, 293 (1987).

[34] J. Pipin and D. M. Bishop, Phys. Rev. A 45, 2736 (1991).

[35] P. S. Julienne, J. Res. Natl. Inst. Stand. Technol. 101, 487 (1996).

[36] E. R. I. Abraham, W. I. McAlexander, J. M. Gerton, R. G. Hulet, R. R. Côté, and A. Dalgarno, Phys. Rev. A 55, R3299 (1996).

[37] A. N. Nikolov, E. E. Eyler, X. T. Wang, J. Li, H. Wang, W. C. Stwalley, and P. L. Gould, Phys. Rev. Lett. 82, 703 (1999).

[38] http://lasim.univ-lyon1.fr/perso/allouche/index.html

[39] C. Amiot, J.Vergés, and C. E. Fellows, J. Chem. Phys. 103, 3350 (1995).

[40] H. Wang, P. L. Gould, and W. Stwalley, J. Chem. Phys. 106, 7899 (1997).

[41] C. J. Williams, E. Tiesinga, P. S. Julienne, H. Wang, W. C. Stwalley, and P. L. Gould, Phys. Rev. A 60, 4427 (2000).

[42] T. Loftus, C. A. Regal, C. Ticknor, J. L. Bohn, and D. S. Jin, Phys. Rev. Lett. 88, 173201 (2002).

[43] C. A. Regal and D. S. Jin, Phys. Rev. Lett. 90, 230404 (2003).

[44] R. Côté and A. Dalgarno, Phys. Rev. A 58, 498 (1998).

[45] R. Côté, A. Dalgarno, Y. Sun, and R. G. Hulet, Phys. Rev. Lett. 74, 3581 (1995).

[46] E. R. I. Abraham, W. I. McAlexander, J. M. Gerton, R. G. Hulet, R. R. Côté, and A. Dalgarno, Phys. Rev. A 53, R3713 (1996).

[47] E. L. Bolda, E. Tiesinga, and P. S. Julienne, Phys. Rev. A 68, 032702 (2003).

[48] E. L. Bolda, E. Tiesinga, and P. S. Julienne, Phys. Rev. A 66, 013403 (2002).

[49] W. Salzmann, U. Poschinger, R. Wester, M. Weidemuller, A. Merli, S. M. Weber, F. Sauer, M. Plewicki, F. Weise, A. M. Esparza, et al., Phys. Rev. A 73, 023414 (2006).

[50] B. L. Brown, A. J. Dicks, and I. A. Walmsley, Phys. Rev. Lett. 96, 173002 (2006).

[51] C. P. Koch, R. Kosloff, and F. Masnou-Seeuws, Phys. Rev. A 73, 043409 (2006).

[52] T. Takekoshi, J. R. Yeh, and R. J. Knize, Opt. Commun. 114, 421 (1995).

[53] M. Mérawa and M. Rérat, Eur. Phys. J. D 17, 329 (2001).

[54] S. Magnier and M. Aubert-Frécon, J. Quant. Spec. Rad. Trans. 75, 121 (2002).

[55] F. A. van Abeelen, D. J. Heinzen, and B. J. Verhaar, Phys. Rev. A 57, R4102 (1998).

[56] P. Courteille, R. S. Freeland, D. J. Heinzen, F. A. van Abeelen, and B. J. Verhaar, Phys. Rev. Lett. 81, 69 (1998). 